\title{
The Cross-Section of Investing Skill
}

\author{
Ravindra Sastry
}

Submitted in partial fulfillment of the requirements for the degree of Doctor of Philosophy under the Executive Committee of the Graduate School of Arts and Sciences

\section{COLUMBIA UNIVERSITY}

2012 
(c) 2012

Ravindra Sastry

All Rights Reserved 


\section{ABSTRACT}

\section{The Cross-Section of Investing Skill}

\section{Ravindra Sastry}

Building on insights from the economics of superstars, I develop an efficient method for estimating the skill of mutual fund managers. Outliers are especially helpful for disentangling skill from luck when I explicitly model the cross-sectional distribution of managerial skill using a flexible and realistic function. Forecasted performance is dramatically improved relative to standard regression estimates: an investor selecting (avoiding) the best (worst) decile of funds would improve risk-adjusted performance by $2 \%(3 \%)$ annually. The distribution of skill is found to be fat-tailed and positively skewed, providing a theoretical explanation for the convexity of fund flows. 


\section{Table of Contents}

I Motivation and Model 1

1 Introduction 2

2 Model 6

2.1 Background ......................... 6

2.2 Model specification . . . . . . . . . . . . . . . . 8

2.3 Bayesian approach . . . . . . . . . . . . . . . 9

2.4 Prior specification . . . . . . . . . . . . . . . . . . . 10

2.5 Hierarchical structure . . . . . . . . . . . . . . . . . . . 12

II Empirics 13

3 Data $\quad 14$

3.1 Multiple share classes . . . . . . . . . . . . . . . . . 15

3.2 Fund objectives . . . . . . . . . . . . . . . . 16

3.3 Fund managers . . . . . . . . . . . . . . . . . . 17

$\begin{array}{llr}4 & \text { Results } & 19\end{array}$

4.1 Parameters of the mixture-of-normals distribution . . . . . . . . . . 20

4.2 Comparison of skill distribution moments, by prior . . . . . . . . . 21

4.3 Estimated cross-sectional distribution of alpha . . . . . . . . . . 25 
4.4 Decile portfolio performance . . . . . . . . . . . 26

4.5 Fund-by-fund alpha correlations . . . . . . . . . . . . . . . . . 31

5 Robustness Checks 33

5.1 Incubation . . . . . . . . . . . . . . . . . . . . . 33

5.2 Alternative Alpha Distribution _. . . . . . . . . . . . . . 34

5.3 Non-normal Idiosyncratic Errors . . . . . . . . . . . . . . . . . . 41

5.4 Alternative Ranking Rules . . . . . . . . . . . . . . . . . . . . . . . 42

5.5 Alternative Performance Measures . . . . . . . . . . . . . . . . . . . . 44

5.5.1 Decile Portfolio Performance . . . . . . . . . . . . . . . . . 44

5.5.2 Fund-Level Return Predictability . . . . . . . . . . . . . . . . 46

III Discussion $\quad 51$

6 Implications $\quad 52$

6.1 Convexity of fund flows . . . . . . . . . . . . . . . 53

6.2 Predictability of fund flows . . . . . . . . . . . . 56

6.3 Smart money effect . . . . . . . . . . . . . . . . . . 60

6.4 Value of active management . . . . . . . . . . . . . 62

$\begin{array}{lll}7 & \text { Conclusion } & 65\end{array}$

IV End Matter $\quad 66$

A MCMC Algorithm: Mixture of Normals $\quad 67$

A.1 Parameters to be estimated . . . . . . . . . . . 67

A.2 Latent indicator . . . . . . . . . . . . . . . . 67 


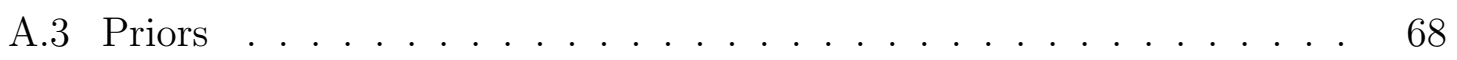

A.4 Sampling algorithm . . . . . . . . . . . . . . . 68

B Moments of the Generalized Unimodal Distribution 72

$\begin{array}{ll}\text { Bibliography } & 73\end{array}$ 


\section{List of Figures}

3.1 Levenshtein distances of all pairs of fund names . . . . . . . . . . . 16

3.2 Number of funds in the sample before and after the share class filter . 17

3.3 Effect of requiring fund manager information on sample size . . . . . 18

4.1 Rolling posterior estimates and 95\% confidence intervals for the means and weights of both mixture components in $(2.2) \ldots \ldots 22$

4.2 Rolling posterior estimates and 95\% confidence intervals for the standard deviations of both mixture components in (2.2) . . . . . . . . 23

4.3 Comparison of the moments of the distribution of skill over time, by model ........................... 24

4.4 Overall estimated cross-sectional distribution of alpha from 2004 . . . 26

4.5 Estimated mixture components from 2004 . . . . . . . . . . . . . 27

4.6 Tail behavior in the cross-sectional distribution of alpha from 2004 . . 27

4.7 Out-of-sample OLS alphas and 95\% confidence intervals for decile portfolios, by ranking method, 1961-2010 . . . . . . . . . . . . 30

5.1 Out-of-sample OLS alphas and 95\% confidence intervals for decile portfolios, after applying age filter, by ranking method, 1967-2010 . . . . 36

5.2 Family of curves generated by (5.1), showing symmetric Student's $t$ distribution $(\gamma=1)$ and two positively skewed counterparts $(\gamma=2$,

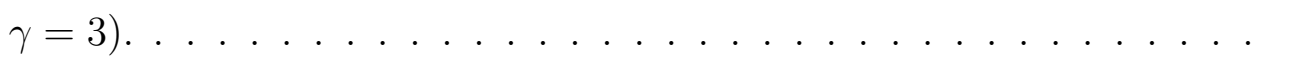


5.3 Family of curves generated by (5.1), showing a positively skewed distribution and the corresponding negatively skewed distribution. . . . 38

5.4 Out-of-sample monthly alphas of decile portfolios formed using alternative alpha distribution, $1963-2010 \ldots \ldots$. . . . . . . . 40

5.5 Histograms of fund-level residual skewness, computed according to (5.3) and (5.4), for nine non-overlapping 24-month intervals, 1992-2009. 43

5.6 Scatterplot showing monthly $\rho_{t}^{\text {mixture }}$ versus $\rho_{t}^{O L S}, 1963-2010 \ldots \ldots . .49$

5.7 Distribution of correlation gap, $\delta_{t}$, as defined in (5.7), 1963-2010 . . . 50

6.1 Updating rules for managerial skill with a mixture-of-normals prior and a normal prior with matching first and second moments . . . . 55

6.2 Estimated fraction of managers with positive alpha using the Bayesian mixture model. . . . . . . . . . . . . . . . . . . 62 


\section{List of Tables}

4.1 Out-of-sample monthly alphas of decile portfolios by ranking method,

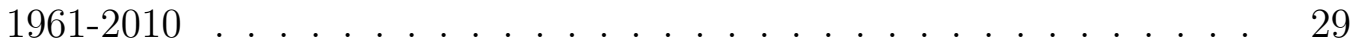

4.2 Serial correlations of estimated fund alphas . . . . . . . . . . . 32

5.1 Out-of-sample monthly alphas of decile portfolios, after age filter, by ranking method, $1967-2010 \ldots \ldots . \ldots . \ldots . \ldots 35$

5.2 Out-of-sample monthly alphas of decile portfolios formed using alternative alpha distribution, $1963-2010 \ldots \ldots 39$

5.3 Out-of-sample monthly alphas of decile portfolios sorted on t-statistics, by ranking method, $1963-2010 \ldots \ldots \ldots$. . . . . . . . . . 45

5.4 Mean excess returns of decile portfolios, by ranking method, 1963-2010 47

5.5 Annualized Sharpe ratios of decile portfolios, by ranking method, 1963-

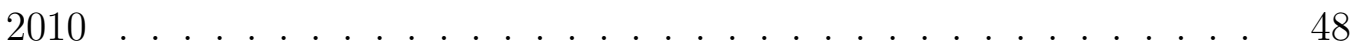

6.1 Results for Fama-MacBeth regressions predicting flow ratio, defined as this month's net flows divided by last month's total assets under management; 1963-2010 .................. 58

6.2 Results for Fama-MacBeth regressions predicting flow ratio using "skill" and "luck" decomposition; 1963-2010 . . . . . . . . . . . . . . . 59

6.3 Results for Fama-MacBeth regressions predicting monthly fund re-

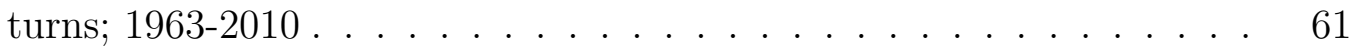




\section{Acknowledgments}

I am grateful to Tom Kane and Don Morrison for putting me on this path, and to the members of my committee - Wei Jiang, Lars Lochstoer, Wes Gray, and especially Mike Johannes and Paul Tetlock — for helping me to come this far.

I am deeply indebted to Bjarni Torfason, my brother-in-arms and a friend for all seasons. Thanks also to my other Ph.D. colleagues for their help and encouragement: Bronson Argyle, Jun Kyung Auh, Mattia Landoni, Zhongjin Lu, and Fangzhou Shi.

I thank seminar participants at Columbia University, the Federal Reserve Board of Governors, Indiana University, Southern Methodist University, the University of Houston, the University of Melbourne, the University of Miami, and Vanderbilt University for their helpful comments. 
For Amber. 


\section{Part I}

Motivation and Model 


\section{Section 1}

\section{Introduction}

With over $\$ 3$ trillion in aggregate assets under management, the scale of the active mutual fund industry is a testament to the conviction among investors that skilled managers exist and can be identified in advance. Yet the unpredictability of good performance remains the principal stylized fact in the mutual fund literature, and is often interpreted to imply the absence of skill among fund managers and the inability of investors to benefit from active mutual funds. ${ }^{1}$ In this paper, I will show that this "fact" is the consequence of strong, unrealistic, and often implicit assumptions about the distribution of "alpha" — namely that alphas are independent across funds, or that alpha is normally distributed. I propose a hierarchical model of investment performance that relaxes both assumptions, and I provide empirical support for alternative "facts": alphas are predictable, and extreme performance is not always luck.

The assumption that alphas are independent across funds is what justifies any fund-by-fund approach to performance evaluation. ${ }^{2}$ Although seemingly reasonable, Jones and Shanken (2005) show that this is equivalent to assuming, counterfactually, that the distribution of alphas across managers is known. In the realistic case where

\footnotetext{
${ }^{1}$ Berk and Green (2004) propose a model that reconciles unpredictability with the existence of skill, but all benefits from skill accrue to fund managers rather than their investors.

${ }^{2}$ Carhart (1997) uses OLS, but more recent methods allow substantially improved inference. See, e.g., Pástor and Stambaugh (2002), Bollen and Busse (2005), and Mamaysky et al. (2007).
} 
the distribution of alphas is acknowledged to be unknown, the returns of all funds intuitively help to illuminate the abilities of fund managers as a group, which in turn enables improved inference for any particular fund. Jones and Shanken present a hierarchical model that formalizes this cross-sectional "learning across funds".

They also impose normality on the cross-sectional distribution of alpha, but there is little evidence that alpha follows this convenient form. ${ }^{3}$ Rather, it is a modeling choice intended to improve inference by reducing the influence of outliers. When extreme alpha estimates are thought to be spurious, reasonable remedies include shrinkage towards a normal distribution, or still more cautious parameter filters that simply drop funds with estimated alphas outside some allowable range. ${ }^{4}$ The surviving funds' alphas can be interpreted with greater confidence, but little is learned about the dropped funds. This is a disadvantage of employing such sharp, and inherently subjective, parameter filters. But the larger cost of treating outliers as problematic statistical artifacts is that the extreme alphas might instead be the most interesting and informative cases - economic rather than statistical outliers.

The "superstars" literature documents convex returns to talent across a range of disciplines. Rosen (1981) describes the phenomenon: "In certain kinds of economic activity there is concentration of output among a few individuals, marked skewness in the associated distributions of income, and very large rewards at the top." Subsequent work explores the pronounced disparities in, for example: CEO compensation, rock concert revenues, television ratings for NBA games, and citation counts for fi-

\footnotetext{
${ }^{3}$ Kosowski et al. (2006) and Fama and French (2010) employ distinct bootstrap techniques and document non-normalities in the alpha distribution. Kosowski et al. find more pronounced nonnormalities than do Fama and French. They also find stronger evidence of positive alphas net of fees.

${ }^{4}$ Mamaysky et al. (2007) use a Kalman filter to estimate alphas and market betas, and then drop funds where $\left(\widehat{\alpha}, \widehat{\beta}_{m k t}\right)$ falls outside a predefined region.
} 
nance academics. ${ }^{5}$ Across diverse fields of endeavor, observed outcomes are distinctly non-normal and positively skewed, regardless of the distribution of underlying, and unobservable, talent. Viewed through this lens, extreme alphas are the natural result of differences in innate investment skill.

This perspective requires some semantic clarification. Alpha is often considered synonymous with investing skill, but these are distinct concepts. Investment skill itself - like the related attribute of intelligence - cannot be directly observed, although it is associated with observable manager characteristics. ${ }^{6}$ Alpha is a measure of investment performance, an outcome that we expect to be increasing in managerial skill. The economics of superstars shows that the mapping between talent and outcomes is often convex, and that extreme alphas may be the rule rather than the exception. The approach to performance evaluation in this paper incorporates these insights. ${ }^{7}$ (For the sake of consistency with the mutual fund literature, alpha and skill are used interchangeably in the rest of this paper.)

I present a hierarchical model where the cross-sectional distribution of alpha is described by a mixture-of-normals, and each fund manager represents a draw from this distribution. The mixture-of-normals is a flexible and realistic modeling choice, allowing - but not imposing - both skewness and fat tails. The data will dictate whether these features are present.

Out-of-sample tests reveal significant short-term alpha predictability. The "mixture" alphas are striking improvements over the estimates from OLS, which in turn outperform alphas from a hierarchical model with a normal cross-sectional distribu-

\footnotetext{
${ }^{5}$ These four examples are from Gabaix and Landier (2008), Krueger (2005), Hausman and Leonard (1997), and Chung and Cox (1990), respectively.

${ }^{6}$ See, e.g., Chevalier and Ellison (1999), Cohen et al. (2008), and Coval and Moskowitz (2001) who relate managerial performance to undergraduate institution quality, social ties to corporate board members, and geographic proximity, respectively.

${ }^{7}$ The distinction between alpha and skill is also central to the model of Berk and Green.
} 
tion: imposing the wrong structure on the cross-section of alpha can be worse than imposing no structure at all. The bottom decile portfolio of mutual funds formed using the mixture-of-normals distribution is more than twice as bad as the OLS bottom decile portfolio, while the top "mixture" portfolio is nearly twice as good as the OLS top portfolio. (The alphas of the top and bottom "mixture" decile portfolios are $+5.4 \%$ and $-6.1 \%$, respectively.) I find that the true distribution of alpha is fat-tailed and positively skewed, consistent with the economics of superstars. I also find that fund flows are sensitive to these "mixture" alphas - evidence that investors respond to, and benefit from, the skills of mutual fund managers. Finally, I show that the non-normality of the alpha distribution can help to explain the convexity of fund flows. ${ }^{8}$

The rest of the paper proceeds as follows. Section 2 describes the hierarchical model in detail. Section 3 explains the data used for the analysis. Section 4 presents the main empirical results. Section 5 provides some robustness checks. Section 6 discusses the results and their relation to the mutual fund literature. Section 7 concludes.

\footnotetext{
${ }^{8}$ Fund flows are much more responsive to high performance than to poor performance.
} 


\section{Section 2}

\section{Model}

I adopt a hierarchical Bayesian model, similar in spirit to Jones and Shanken (2005), that allows for skewness and fat-tails in the cross-sectional distribution of managerial skill. The parameters of this distribution are determined by the data, including "outliers". Skill is measured relative to a four-factor model including the Fama and French (1993) factors and the momentum factor of Carhart (1997). Prior distributions are weakly informative-proper but diffuse.

\subsection{Background}

The (semi-strong) efficient markets hypothesis implies that abnormal mutual fund returns should not persist. Carhart (1997) provides strong supporting evidence: adjusting for a momentum factor in addition to the three Fama-French (1993) factors produces ranked portfolios of mutual funds without significant persistence among top performing funds. However, Berk and Green (2004) showed that if fund returns are decreasing functions of assets under management, the absence of persistence in factormodel skill estimates is not inconsistent with the existence of skill. ${ }^{9}$ Furthermore, studies using shorter holding periods than the one-year holding periods employed by

\footnotetext{
${ }^{9}$ Berk and Green (2004) also assume rational investor learning in the context of a commonknowledge prior on the distribution of skill. This is an unrealistic feature of their model.
} 
Carhart have found evidence of persistence. ${ }^{10}$ Skill may indeed exist, but it appears difficult to identify.

Imperfect empirical methodologies may explain the difficulty. Jones and Shanken (2005) observe that "... the maximum OLS alpha estimate [among a population of funds] becomes unbounded as the number of funds approaches infinity, even when the true alphas are all zero." 11 Mamaysky et al. (2007) demonstrate that "... sorting on the estimated alphas populates the top and bottom deciles not with the best and worst funds, but with those having the greatest estimation error." Carhart's result appears less damning upon reconsideration: alpha may seem non-persistent simply because it is poorly measured.

One "solution" is to drop funds with suspiciously extreme alphas. Another approach is to use shrinkage estimators. ${ }^{12}$ Bayesian inference automatically incorporates shrinkage towards a prior distribution. Inferences are made on all funds, but the accuracy of the posterior estimates depends very strongly on the shape of the prior.

Baks et al. (2001) employ a prior on skill consisting of the right tail of a truncated normal distribution and a probability mass at the point of truncation. They provide a procedure for eliciting the parameters of this prior from the investor. Jones and Shanken use a normal prior on skill but do not specify its mean or variance, instead estimating these along with all fund-level parameters in a hierarchical procedure. Both priors are simple, but they do not reflect the richness of the true distribution of skill.

Kosowski et al. (2006) document a complex non-normal distribution of mutual fund alphas. They note that "the separation of luck from skill becomes extremely

\footnotetext{
${ }^{10}$ See, e.g., Bollen and Busse (2004).

${ }^{11}$ They assume a standard factor model with independent residuals, as in (2.1).

${ }^{12}$ See Efron and Morris (1977) for an introduction to shrinkage estimation.
} 
sensitive to the assumed joint distribution from which the fund alphas are drawn" and motivate their non-parametric bootstrap approach by invoking the "intractability of parametrically modeling the joint distribution of mutual fund alphas across several hundred funds." This paper tackles the same problem head-on. The prevailing crosssectional distribution of skill among mutual fund managers is indeed complex, but it is not intractable.

\subsection{Model specification}

Excess fund returns are assumed to follow a standard linear factor structure,

$$
R_{t}^{j}-R_{t}^{f}=\alpha^{j}+F_{t} \beta^{j}+\varepsilon_{t}^{j}
$$

where $\varepsilon_{t}^{j} \sim N\left(0, V_{\varepsilon}^{j}\right)$, factor loadings $\beta^{j}$ and residuals $\varepsilon^{j}$ are cross-sectionally uncorrelated, and the $T \times K$ matrix of factor returns $F$ is observable.

The investor assumes that true manager alphas are independent draws from a finite mixture of two normal distributions, with density

$$
f\left(\alpha^{j}\right)=\sum_{i=1}^{N} w_{i} \cdot \frac{1}{\sqrt{V_{i}}} \cdot \phi\left(\frac{\alpha^{j}-\mu_{i}}{\sqrt{V_{i}}}\right)
$$

where $N=2, \phi(x)$ is the standard normal density, $\mu_{i}$ and $V_{i}$ are the unknown mean and variance of mixture component $i$, and the $w_{i}$ are unknown non-negative weights that sum to one. ${ }^{13}$ This conditional distribution of alpha is simple and flexible, allowing both skewness and excess kurtosis in the cross-section of managerial skill

\footnotetext{
${ }^{13}$ Without additional restrictions, the mixture of normals in (2.2) is not identified. An additional identifying restriction, $\mu_{2} \geq \mu_{1}$, is imposed without loss of generality to allow estimation. Stephens (2000) describes the problem in more detail and proposes an alternative resolution.
} 
but imposing neither. ${ }^{14}$ It also suggests an intuitive interpretation of the two mixture components as "good" and "bad" manager types.

Greater flexibility could be achieved at the expense of parsimony. Increasing the number of normal mixture components is straightforward, although interpretation is problematic for $N \geq 4$. One could alternately employ finite mixtures of Student's $t$ or Laplace distributions - imposing excess kurtosis - or a more general unimodal skewed distribution. ${ }^{15}$

In the absence of strong justification for any specific alternative distribution, however, it is reasonable to begin with the simplest choice that allows some variability in skewness and kurtosis. The distribution in (2.2) fits the bill, while minimizing computational complexity and the impact of specification searching.

\subsection{Bayesian approach}

The hierarchical model defined by (2.1) and (2.2) is conceptually simple, yet estimation with classical methods is not feasible. Consider the sheer number of parameters: for each of the $J$ funds there are $K+2$ parameters, in addition to 5 population-level hyper-parameters. ${ }^{16}$ For any reasonable values of $J$ and $K$, the prospects for successfully implementing a high-dimensional numerical optimization, as required by MLE or GMM, are dim.

\footnotetext{
${ }^{14}$ The distribution is conditional because it depends on the unknown population hyper-parameters $\left(\mu_{i}, V_{i}, w_{i}\right)$ that will be estimated along with the fund-level parameters $\left(\alpha^{j}, \beta^{j}, V_{\varepsilon}^{j}\right)$.

${ }^{15}$ Fernández and Steel (1998) describe a general approach to modeling skewed unimodal distributions in a Bayesian context. Their method allows skewness and kurtosis to vary independently; an advantage over the simpler model in (2.2). See section 5.2 for an application of their method to this analysis.

${ }^{16}$ The $K+2$ parameters are the managerial skill and the idiosyncratic variance, in addition to $K$ factor loadings.
} 
The Markov chain Monte Carlo (MCMC) approach to the estimation of Bayesian models breaks this curse of dimensionality. Rather than dealing directly with a single intractable $[J \cdot(K+2)+5]$-dimensional joint posterior distribution, we can equivalently work with the $[J \cdot(K+2)+5]$ individual 1-dimensional conditional posterior distributions, which together fully characterize the joint posterior distribution. This equivalence is guaranteed by the Hammersley-Clifford theorem. ${ }^{17}$

\subsection{Prior specification}

A complete Bayesian model requires both likelihoods and priors. The former are defined by (2.1) and (2.2). The latter are described below.

An ideal prior distribution (i) accurately represents the investor's prior information and beliefs while (ii) enabling easy sampling from the posterior distribution. The second condition is often automatically satisfied by a conjugate prior, which gives rise to a known analytical posterior distribution from the same family as the prior. For example, a normal prior and a normal likelihood result in a normal posterior. Conjugacy is a valuable property, but is not always consistent with (i). In such cases, I relax (ii) in favor of (i).

A common criticism of Bayesian inference is that the inherently subjective prior distribution can exert substantial influence on posterior parameter estimates. I minimize subjectivity by choosing intentionally uninformative priors. By this, I mean

\footnotetext{
${ }^{17}$ See, e.g., Robert and Casella (2004).
} 
diffuse proper distributions. These are sometimes also called weakly informative priors to distinguish them from improper priors. ${ }^{18}$

Putting aside complex approaches to uninformative priors that are themselves subject to criticism, ${ }^{19}$ I follow the pragmatic lead of Leamer (1978):

"... we can find a prior distribution that will have little impact on the posterior. It is important to understand that this is not a prior intended to represent ignorance... In practice, the sample may dominate the prior information and the posterior distribution may be inconsequentially different from a posterior distribution corresponding to an improper [uninformative] prior. A prior density that is relatively uniform where the likelihood function attains its maximum is likely to imply such a posterior." 20

The priors on $\mu_{i}$ and each $\beta_{k}^{j}$ are independent normal distributions with means of zero and large variances, with the exception of $\beta_{m k t}^{j}$ which has a prior mean of one. The priors on $V_{i}$ and $V_{j}^{\varepsilon}$ are independent gamma distributions with shape parameters of one and large scale parameters. ${ }^{21}$ The prior on $w=\left[w_{1}, \ldots, w_{N}\right]$ is a Dirichlet distribution with an $N \times 1$ vector of ones as the parameter. ${ }^{22}$ The prior on $\alpha^{j}$ is

\footnotetext{
${ }^{18}$ Any valid probability density must have a finite integral over the relevant parameter space. An improper prior is a function whose integral is infinite, hence the impropriety. Typical improper priors would be $f(\theta) \propto 1$ for a location parameter, or $f(\sigma) \propto \frac{1}{\sigma}$ for a scale parameter. While superficially appealing, such priors do not always lead to valid posteriors. (See, e.g., Lindley (1972).) They are not even uninformative. The improper prior $f(\theta)$ appears to be uninformative with respect to $\theta$, but it also suggests that $|\theta|$ is extraordinarily large and that $\frac{1}{\theta}$ is very close to zero.

${ }^{19}$ For example, the Jeffreys prior or Zellner's $g$-prior.

${ }^{20}$ pp. 61-63, emphasis added.

${ }^{21} \mathrm{~A}$ more common choice would be inverse-gamma distributions, which have the virtue of conjugacy. However, these are not consistent with condition (i) or Leamer's rule of thumb, especially when the posterior parameter estimates are likely to be close to zero. See Gelman (2006).

${ }^{22}$ This results in a marginal prior for each $w_{i}$ that is beta distributed with parameters 1 and $N-1$. For the degenerate case where $N=2$ that is employed here, this is simply the uniform distribution on the unit interval.
} 
already defined by (2.2). See Appendix A for a detailed presentation of the prior and posterior distributions for all model parameters.

\subsection{Hierarchical structure}

Having specified a complete Bayesian model in some detail, it may seem self-defeating to select priors that convey no information. Such a view overlooks the importance of the hierarchical structure of the model. The power of this structure to influence posterior parameter estimates is not undone by arbitrarily diffuse priors, as long as they are proper. ${ }^{23}$ Indeed, the chief justification for choosing diffuse priors is to ensure that the estimation results are driven by the model itself rather than any specific choice of priors.

If we altered the model to eliminate (2.2) and estimated (2.1) with diffuse priors, this would be equivalent to OLS. However, the estimates generated by the full model are radically different from those given by OLS - these differences must be attributed to the hierarchical structure itself.

\footnotetext{
${ }^{23}$ Improper priors are another story, since they can effectively preclude the cross-sectional learning that is integral to hierarchical model estimation.
} 
Part II

\section{Empirics}




\section{Section 3}

\section{Data}

Mutual fund data are obtained from the CRSP Survivor-Bias-Free US Mutual Fund Database, which begins in 1961. Monthly returns are net holding period returns - all estimated alphas are after costs and management fees. ${ }^{24}$ This takes the perspective of an investor considering allocating capital to active fund managers, rather than that of a philosophical investigation of the existence of skill. Alpha only matters to the extent that it exceeds costs.

Monthly factor returns are obtained from Kenneth French's website via WRDS. I use a four-factor model, consisting of the three Fama-French factors and a momentum factor, throughout the analysis.

The CRSP data are incomplete in several key ways. Multiple share classes of a single fund appear as separate funds in CRSP, with no foolproof way of identifying and grouping them. Addressing this shortcoming is especially important in this analysis, since double-counting funds would impair estimation of the cross-sectional distribution of skill. Furthermore, CRSP data regarding fund objectives and fund managers are not comprehensive. Each of these is addressed in turn.

\footnotetext{
${ }^{24}$ To reconstruct gross returns, one should add back management expenses and trading costs. Expense ratios are available for most funds, so these could be added back to the reported net returns. However, trading costs would need to be estimated in order to compute approximate gross returns. See, e.g., Wermers (2000) and French (2008).
} 


\subsection{Multiple share classes}

The cross-sectional distribution of skill in (2.2) can only be estimated if each fund in the sample represents a distinct draw from the distribution. Many mutual funds have multiple share classes, and each class appears in the CRSP database as a separate fund. To proceed with the analysis, these "funds" need to be identified in order to avoid double-counting. Unfortunately, there is no explicit share class variable in the database.

CRSP suggests matching "funds" that share the same portfolio of holdings, but portfolio data are not available until 1998. The only alternative is to examine management company and fund names, matching as deemed appropriate. This latter approach is employed here.

Funds without fund name data are consequently excluded from the analysis. This affects approximately $10 \%$ of the funds at the beginning of the sample in 1961 but generally disallows less than $5 \%$ of all funds.

Fund names of the remaining funds are filtered to remove all non-alphabetic characters and extra spaces. Based on the general pattern that share classes of the same fund will have the same name followed by a suffix denoting the share class, funds are then grouped according to their names' pairwise Levenshtein distances. ${ }^{25}$ Panel A of Figure 3.1 shows a histogram of all pairwise Levenshtein distances for the entire sample of 20,000+ funds. The mode is between 40 and 50, and it appears that very few pairs of funds have similar names. Panel B of Figure 3.1 shows the same histogram only for distances less than or equal to 10 . Here we can see that there is a secondary

\footnotetext{
${ }^{25}$ The Levenshtein distance between two strings is the minimum number of edits needed to transform one string into the other, with the allowable edit operations being insertion, deletion, or substitution of a single character. See Levenshtein (1966).
} 

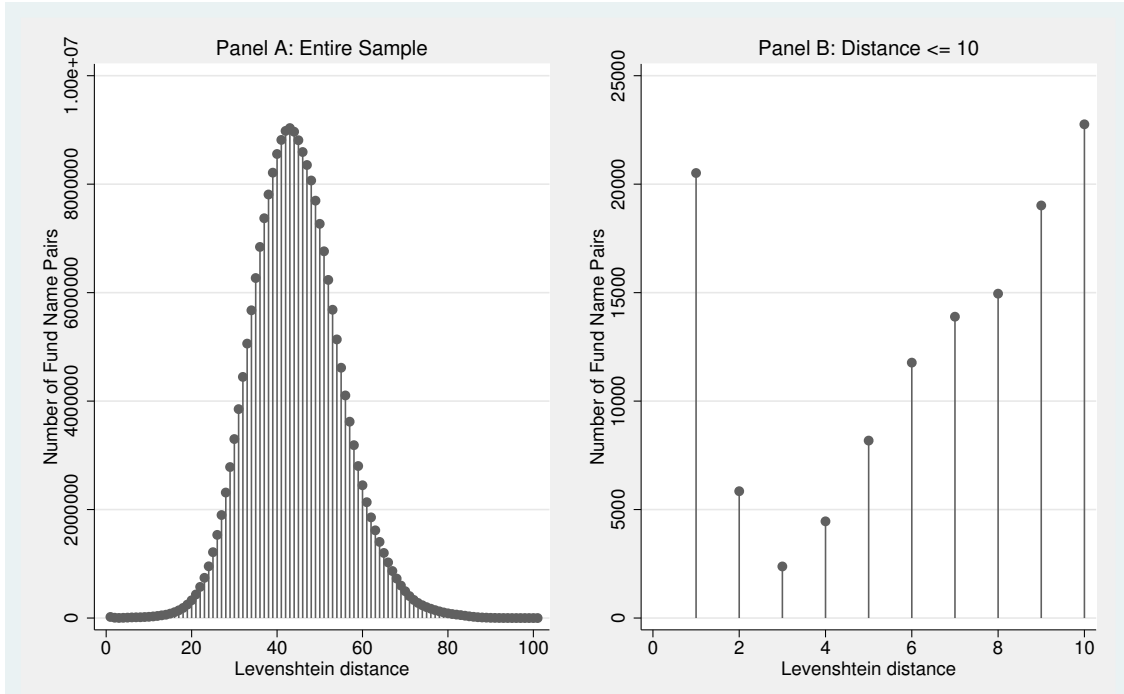

Figure 3.1 - Levenshtein distances of all pairs of fund names. Panel A shows the entire CRSP sample. The mode is quite large - between 40 and 50. Panel B zooms in to show only distances less than or equal to 10, clearly indicating the presence of a secondary mode at 1 .

mode for distances equal to 1 . Although most random pairs of funds have names that are nothing alike, some pairs have names that are nearly identical.

Funds with Levenshtein distances less than 3 are assumed to be share classes of a single actual fund. From each grouping of fund share classes, the class with the most assets under management is retained to represent the fund. Figure 3.2 shows the total number of "funds" in the CRSP database, the number of funds that have fund name data, and the number of true funds that survive the share class filter.

\section{$3.2 \quad$ Fund objectives}

This analysis is confined to actively managed domestic equity mutual funds. Fund names and Lipper class (where available) are screened to drop money market, bond, balanced, and international funds. Index funds are identified by screening fund names for explicit references to indexing. Closet indexers cannot be identified in this way, 


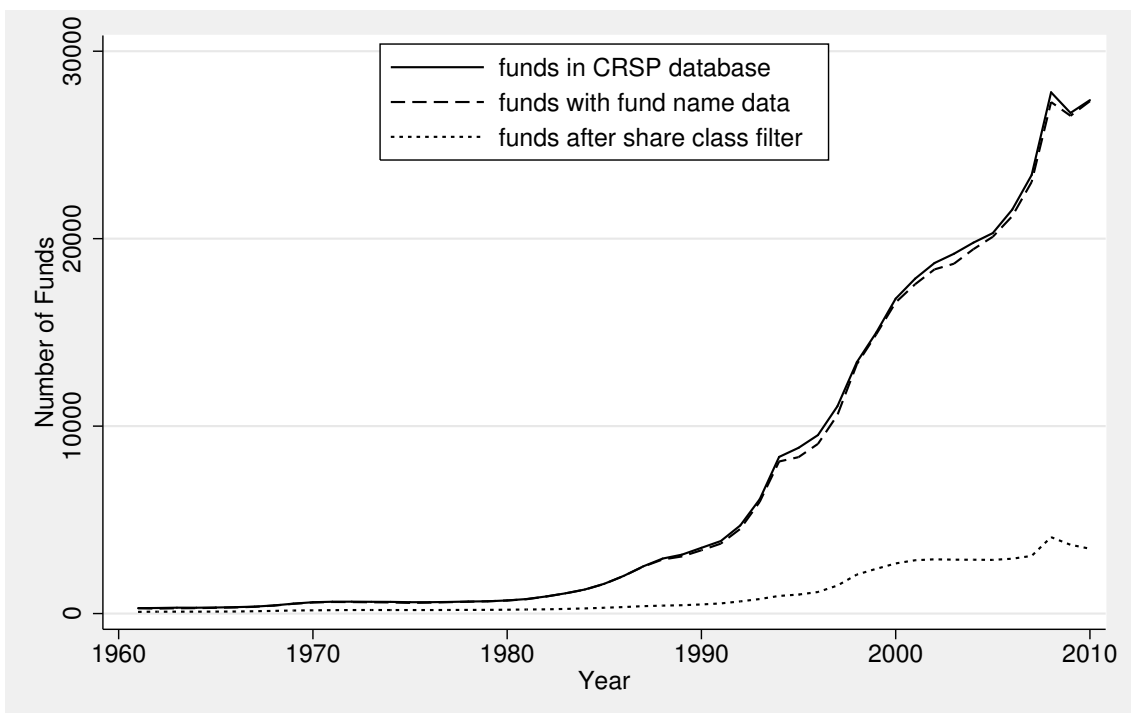

Figure 3.2 - Number of funds in the sample before and after the share class filter. Essentially all funds have fund names recorded in the database (dashed line), but the majority do not survive the share class filter (dotted line).

and any such funds remain in the sample. This corresponds to the challenge faced by investors in prospectively identifying closet indexers. Furthermore, such funds will in general have estimated alphas close to zero, less their trading costs and management fees. They are unlikely to populate the highest or lowest deciles of ranked mutual funds, which are the basis of the key results of this study.

\subsection{Fund managers}

Data on fund managers in the CRSP database are not comprehensive. No manager name data is available before 1998. Even after 1998, in many cases, the fund manager field is missing or recorded as team. ${ }^{26}$ Utilizing individual fund manager data is

\footnotetext{
${ }^{26}$ Massa et al. (2010) discuss why fund management companies may elect to have anonymously managed funds.
} 


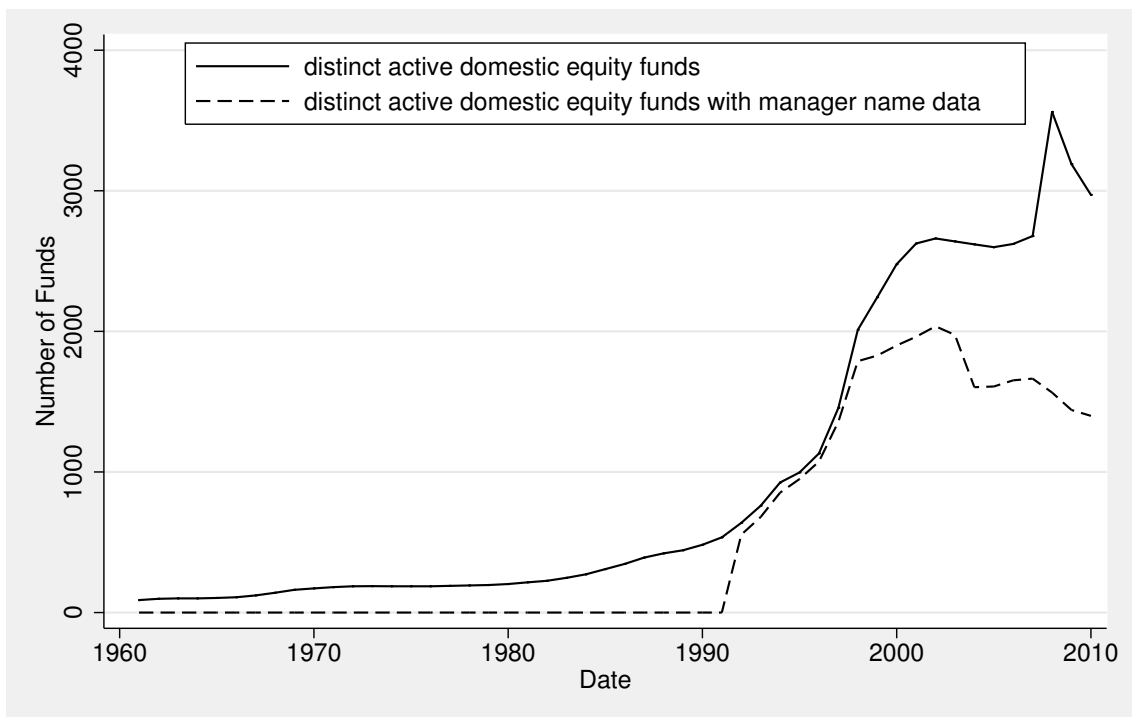

Figure 3.3 - Effect of requiring fund manager information on sample size. The solid line represents the fund sample used in the rest of the analysis. The sudden jump in the number of funds in 2007 corresponds to a change in the data sources used by CRSP.

therefore not feasible without impoverishing the sample. The full impact of requiring individual manager data is depicted in Figure 3.3.

One consequence of the lack of reliable manager data is that the funds themselves become the objects of analysis. Ideally, when a fund changes its portfolio manager, the new prior on its alpha would depend on the new manager's previous fund's returns. Since the data do not allow managers to be tracked in this way, I use funds' returns as if no management changes have occurred. ${ }^{27}$ This does not introduce any bias but does weaken the model's predictive ability for funds that have changed managers during the evaluation period.

\footnotetext{
${ }^{27}$ This approach assumes fund returns are generated according to (2.1) and depend only on the fund manager. If the fund family makes a difference, then future returns are related to past returns even after a managerial change. Brown and Wu (2011) estimate such a model in a Bayesian setting.
} 


\section{Section 4}

\section{Results}

For each month, alphas are estimated for all funds with at least 12 monthly observations during the 2-year window $[t-23, t]$. Funds are evaluated using each of three methods: OLS, hierarchical Bayes with a mixture-of-normals prior, and hierarchical Bayes with a "standard" normal prior. ${ }^{28}$ All funds are ranked according to their estimated alphas and assigned to deciles. Funds that are missing during month $t+2$ are dropped; equally-weighted portfolios are formed from the remaining funds. ${ }^{29}$ These portfolios are held for one month and then rebalanced according to the updated alpha estimates.

Following Busse and Irvine (2006), the alpha of each of these decile portfolios is estimated using standard OLS regressions. When estimating the alpha of a portfolio of funds, there is likely to be little difference between the OLS measure and any reasonable Bayesian measure based on the same model. Using the OLS post-rankingperiod measure avoids any bias that could result from using the Bayesian measure for both the ranking-period and the post-ranking-period.

\footnotetext{
${ }^{28}$ This last method follows Jones and Shanken (2005) and allows a direct assessment of the value of using a sufficiently flexible prior distribution.

${ }^{29} \mathrm{~A}$ one-month gap is introduced between the ranking-period and the post-ranking-period to eliminate spurious microstructure-induced correlations.
} 


\subsection{Parameters of the mixture-of-normals distribution}

Posterior means and 95\% confidence intervals of the parameters in (2.2) are shown in Figure 4.1 and Figure 4.2. Each rolling estimation window is 24 months long, so the estimates depicted for month $t$ correspond to fund performance during the window $[t-23, t]$

Figure 4.1 shows posterior estimates of both mixture component means, $\mu_{1}$ and $\mu_{2}$, and the weight on the second mixture component, $w_{2}$. By construction, and without loss of generality, the mean of the second component is greater than or equal to the mean of the first component. Panel A shows that $\mu_{1}$ is generally indistinguishable from zero, although it does diverge from zero towards the end of the sample - positively during the very early 2000s and negatively since then. Panel B shows that $\mu_{2}$ follows a pattern similar to $\mu_{1}$, essentially always statistically equal to zero. Panel C shows that the proportion of managers drawn from the second, high skill, component has always been low and has steadily decreased over time - falling from $20 \%$ at the start of the sample to $10 \%$ at the end.

Figure 4.2 shows posterior estimates of the standard deviations of both mixture components. Far more managers are drawn from the first component, so the confidence intervals on $\sqrt{V_{1}}$ are much tighter than those on $\sqrt{V_{2}}$. Nevertheless, it is clear that $\sqrt{V_{2}}$ is an order of magnitude larger than $\sqrt{V_{1}}$.

The overall distribution has substantial excess kurtosis because the individual variances are unequal; in this case $V_{2} \gg V_{1}$. This, plus the fact that $\mu_{2}>\mu_{1}$ gives rise to positive skewness as well. Both findings are consistent with the economics of superstars.

Although one virtue of the mixture-of-normals distribution was the potentially straightforward interpretation of the components as good and bad manager types, 
the high dispersion of the second mixture component suggests a similar but more nuanced interpretation. Managers with extremely high or low relative alphas are likely to be drawn from the second mixture component, consistent with positively correlated alpha and risk-taking: managers drawn from this high-alpha, high-variance component are taking aggressive risks that pay off on average but that expose their funds to significant losses.

\subsection{Comparison of skill distribution moments, by prior}

Posterior moments of the distribution of skill for all three "priors" are shown in Figure 4.3. ${ }^{30}$ Moments for both hierarchical Bayes priors - normal and mixture-ofnormals - are computed directly from their posterior parameter estimates. Moments for the OLS distribution are computed from the estimated fund alphas.

Panel A compares the estimated means of the prevailing distribution of skill from OLS and the mixture model. The estimated mean from the normal hierarchical model is nearly identical to that of the mixture model and is not depicted. The posterior mean from the mixture model behaves as we might expect: it generally follows the OLS mean but shrinkage mitigates the larger fluctuations.

Panel B compares the estimated standard deviations of the prevailing distribution of skill. The susceptibility of OLS to the influence of outliers is clear. The normal hierarchical model generates the lowest estimates - shrinkage in its strongest form. In between lies the estimated standard deviation from the mixture hierarchical

\footnotetext{
${ }^{30}$ OLS may not be an explicit Bayesian prior, but OLS estimates will exactly match Bayesian estimates if the priors are dogmatically diffuse; e.g. normal distributions with infinite variances. If such strictly uninformative, improper priors are specified, there can be no shrinkage and the posterior alpha estimates will depend only on the likelihood. Thus, Bayesian estimation would yield the maximum-likelihood estimates for a regression model such as (2.1), which are equivalent to the OLS estimates.
} 


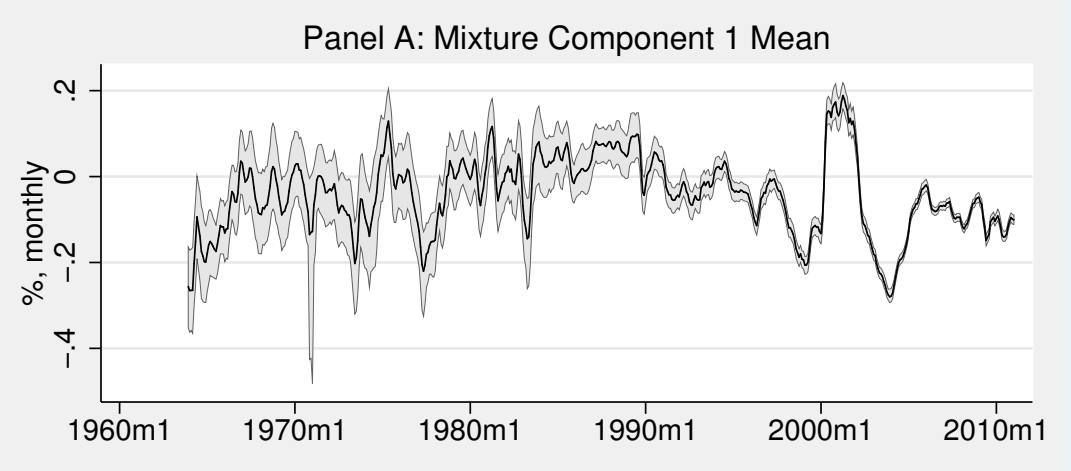

Panel B: Mixture Component 2 Mean

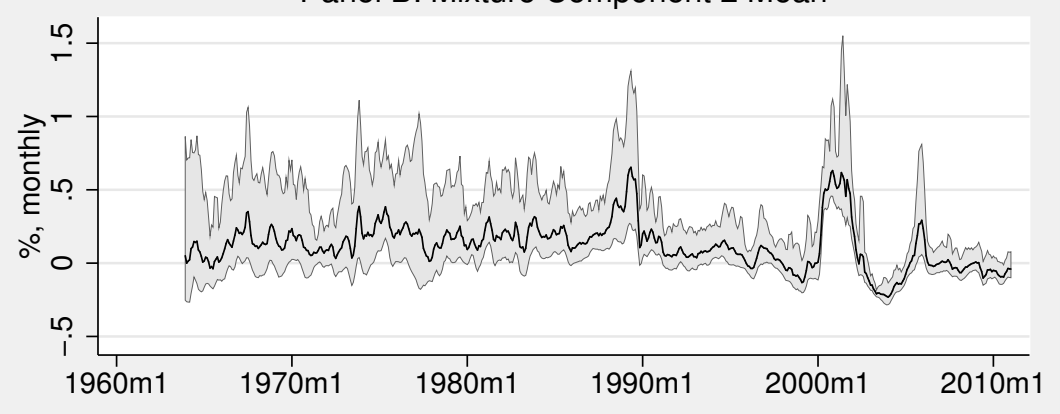

Panel C: Weight on Mixture Component 2

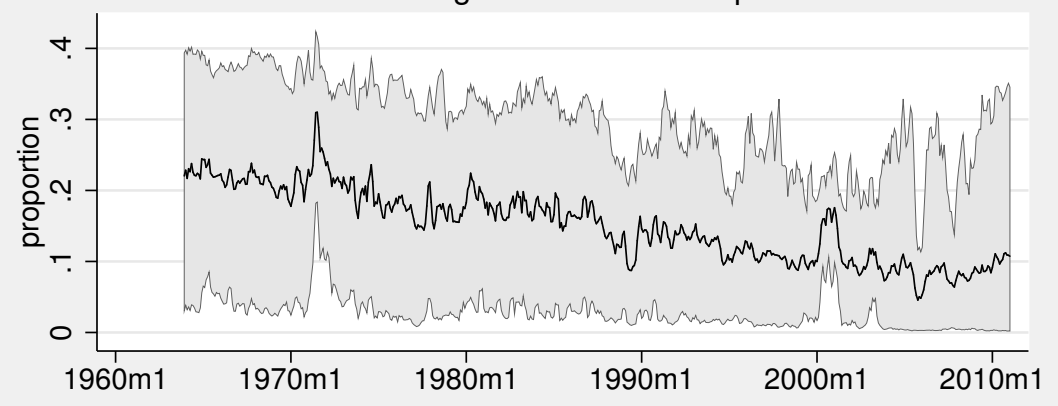

Figure 4.1 - Rolling posterior estimates and 95\% confidence intervals for the means of both mixture components and the weight on the second mixture component in (2.2). The mean of the second component is constrained to be higher than the mean of the second component. 


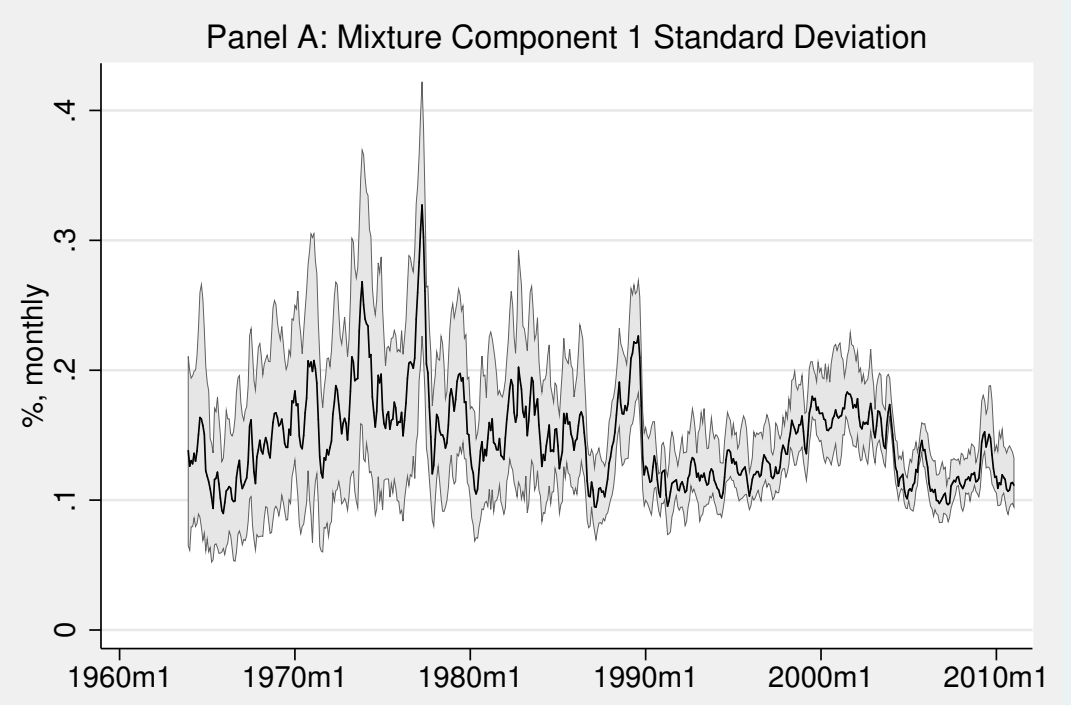

Panel B: Mixture Component 2 Standard Deviation

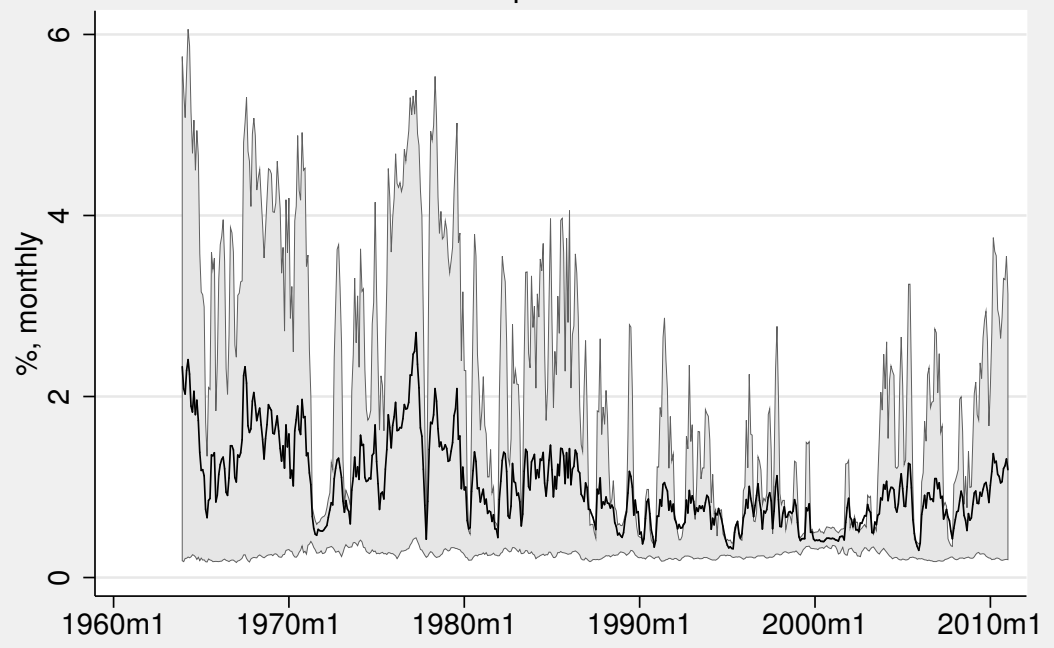

Figure 4.2 - Rolling posterior estimates and $95 \%$ confidence intervals for the standard deviations of both mixture components in (2.2). 


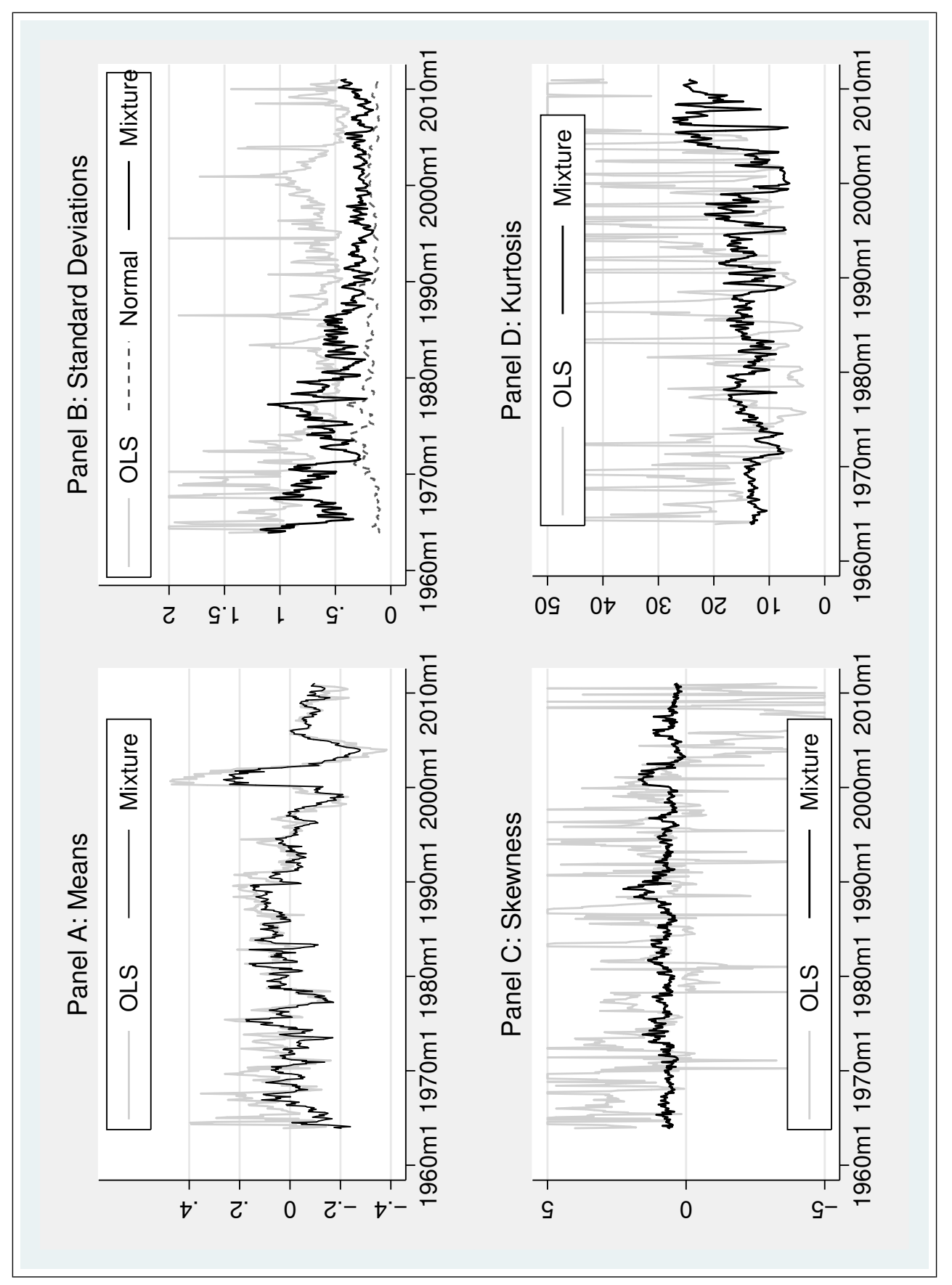

ซี

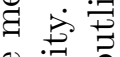
幽 $<$ 嵌 过 ๘ g . 诺 过 s. - 0 ฮे है. 2 م ఉં 0 की

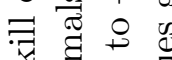
霖 च Н

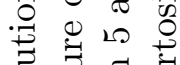

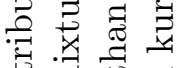
品品 0 † -

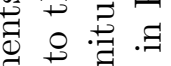
ฮี శ్రి घ. छ

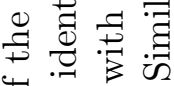

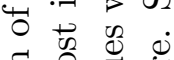

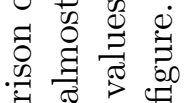
苂 द्व. . की ช ๓. ๑ อำ 00

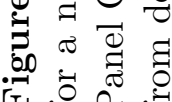


model. This model is flexible enough to take account of extreme alphas without being dominated by them.

Panel C compares the estimated skewness of the prevailing distribution of skill from OLS and the mixture model. The normal model, of course, is constrained to have zero skewness. OLS cannot decide whether skill is positively or negatively skewed. Indeed, the series is so volatile and the excursions from zero are so large (in both directions) that it cannot possibly correspond to reality. The mixture model produces relatively stable and comprehensible estimates of mild positive skewness.

Panel D tells a similar story via the kurtosis estimates. The normal model is constrained to have a kurtosis of three, while both OLS and the mixture model "agree" that kurtosis is likely to be much higher - although they diverge regarding how much higher. Here is clear evidence that the default Bayesian approach, with a normal prior on alpha, over-shrinks the estimated alphas in this case. Fitting to a normal distribution assumes away large outliers, while a more flexible model can learn from them.

\subsection{Estimated cross-sectional distribution of alpha}

The moments of the distribution of alpha are revelatory, but the distribution itself completes the story. The overall distribution of skill is shown in Figure 4.4. This view makes the distribution appear normal, but the pivotal deviations from normality are in the tails.

Understanding the tail behavior requires an examination of the individual mixture components. Both components, scaled to reflect their relative weights, are shown in Figure 4.5. The two components differ dramatically in terms of relative weighting and dispersion. Component 1 represents approximately $85 \%$ of all managers and requires 


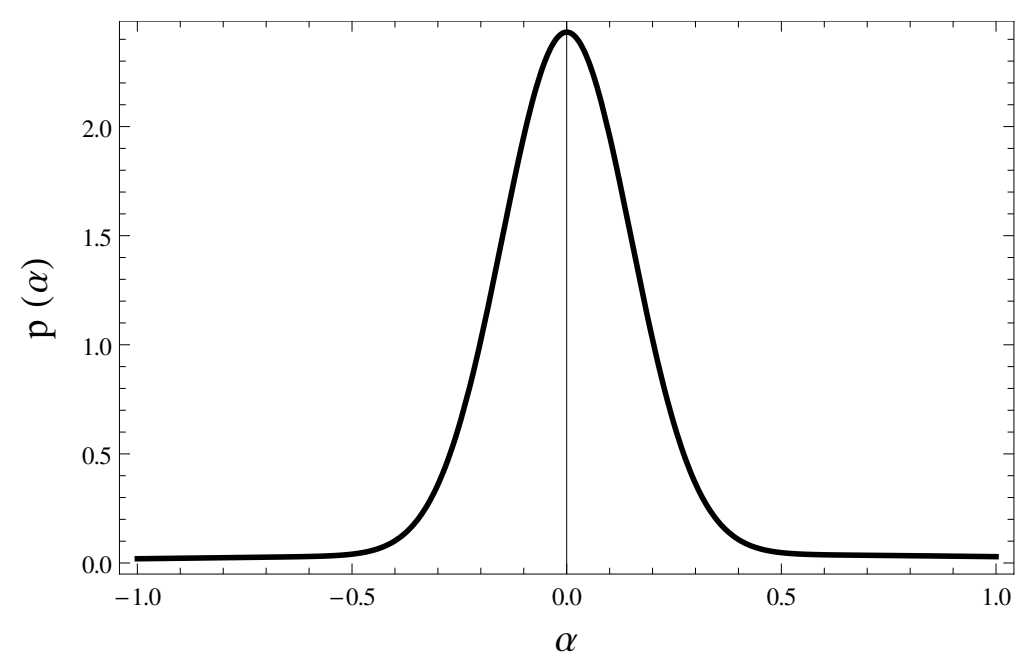

Figure 4.4 - Overall estimated cross-sectional distribution of alpha from 2004, summing the individual components shown in Figure 4.5. Alpha is monthly and measured in percent. The distribution appears generally normal in this zoomed-out view, since the weight on mixture component 1 is much larger than the weight on mixture component 2. A closer examination of the tails, depicted in Figure 4.6, reveals pronounced non-normality.

that alpha is fairly close to zero. Component 2 , representing the remaining $15 \%$ of fund managers, allows for extreme alphas in both directions.

Figure 4.6 illustrates this extreme tail behavior. Although component 1 assigns approximately zero probability to alphas larger than $0.6 \%$ monthly, component 2 assigns small positive probabilities to alphas as high as $3 \%$ monthly. Relative to a normal model of alpha, which completely discounts the possibility of "superstar" fund managers capable of dramatic outperformance, the mixture-of-normals allows the upper echelon of managers to exhibit superior alphas.

\subsection{Decile portfolio performance}

Decile portfolios are formed using all three ranking methods and their abnormal returns are estimated using OLS. The power of the mixture-of-normals prior is made 


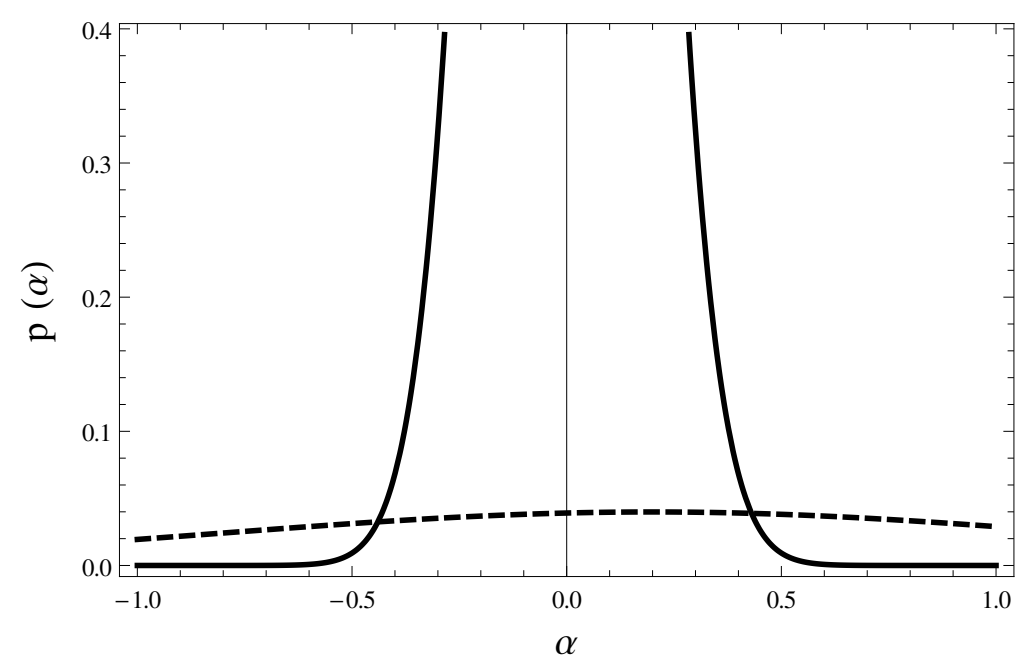

Figure 4.5 - Estimated mixture components from 2004, chosen to be representative. Component 1 is depicted by the solid line, while the higher-mean component 2 is depicted by the dashed line. Alpha is monthly and measured in percent. Component 2 has a slightly higher mean and a much higher variance than component 1 .

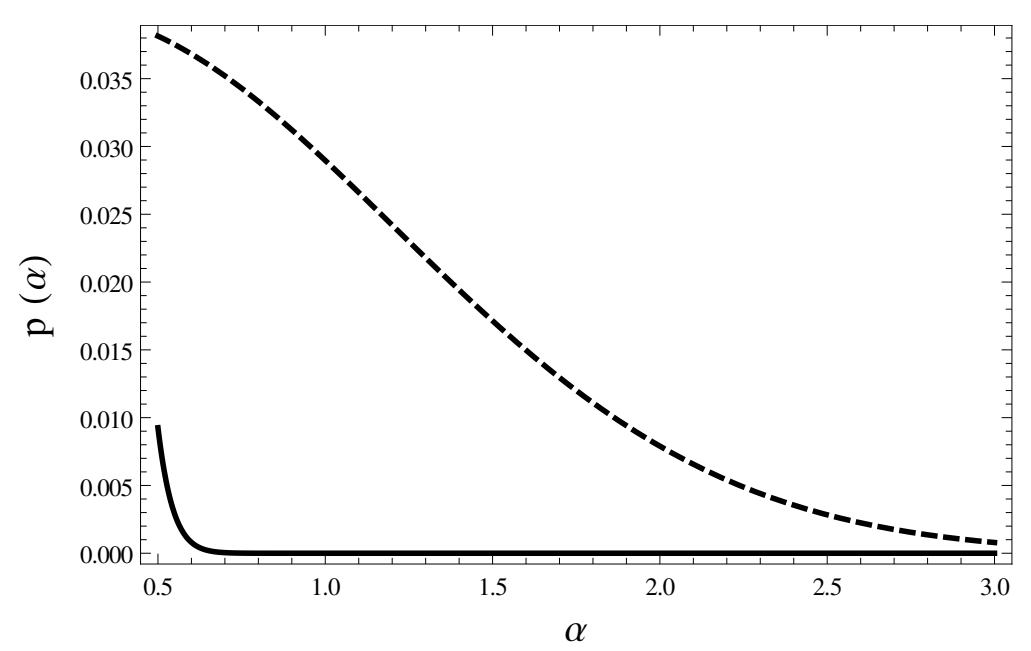

Figure 4.6 - Tail behavior in the cross-sectional distribution of alpha from 2004 - a zoomed-in view of the distributions in Figure 4.5. Component 1 is depicted by the solid line, while the higher-mean component 2 is depicted by the dashed line. Alpha is monthly and measured in percent. The probability of observing extremely high alphas is several orders of magnitude higher than it would be in a model that imposed normality on alpha, a result of allowing for the second component. 
clear in this test. Numerical results are given in Table 4.1 and shown graphically in Figure 4.7.

Even OLS reveals substantial short-term predictability. The worst funds (deciles 1 and 2) continue to under-perform and the best funds (deciles 9 and 10) continue to outperform. Carhart documented persistent poor performance over a longer time frame, attributing it to high fees. Bollen and Busse, among others, have more recently documented persistent outperformance in the short term using OLS. Thus, the results from the OLS decile portfolios are just as expected.

Results using rankings from the normal hierarchical model are telling - they are substantially weaker than those from OLS. The performance of the bottom decile portfolio is better than the OLS bottom decile portfolio, and the performance of the top decile is worse. The normal prior does a worse job of sorting funds. There is valuable information in the extreme alpha estimates that the normal model is forced to disregard.

The results from the mixture hierarchical model are striking: all portfolios have statistically significant alphas, decile 1 under-performs OLS decile 1 by 30 basis points and decile 10 outperforms OLS decile 10 by 18 basis points. When annualized, these translate to 360 basis points and 216 basis points, respectively. The differences relative to the normal hierarchical estimates are even starker. In absolute terms, the mixture decile 1 portfolio posts an annual alpha of $-6.1 \%$ and the mixture decile 10 portfolio posts an annual alpha of $+5.4 \%$ during the fifty-year period 1961-2010. The mixture bottom decile portfolio is more than twice as bad as the OLS bottom decile portfolio, while the mixture top decile portfolio is nearly twice as good as the OLS top decile portfolio. 


\begin{tabular}{cccc}
\hline \multicolumn{4}{c}{$\begin{array}{c}\text { Out-of-Sample Monthly Alphas } \\
\text { of Decile Portfolios }\end{array}$} \\
\hline Decile & OLS & Normal Prior & Mixture Prior \\
\hline 1 & $-0.21^{*}$ & $-0.14^{*}$ & $-0.51^{*}$ \\
& $(0.05)$ & $(0.04)$ & $(0.03)$ \\
2 & $-0.12^{*}$ & $-0.16^{*}$ & $-0.37^{*}$ \\
& $(0.04)$ & $(0.04)$ & $(0.04)$ \\
3 & -0.07 & $-0.09^{*}$ & $-0.34^{*}$ \\
& $(0.04)$ & $(0.04)$ & $(0.04)$ \\
4 & -0.05 & -0.07 & $-0.18^{*}$ \\
& $(0.03)$ & $(0.04)$ & $(0.04)$ \\
5 & -0.03 & -0.02 & $-0.10^{*}$ \\
& $(0.03)$ & $(0.04)$ & $(0.04)$ \\
6 & 0.00 & 0.04 & $0.25^{*}$ \\
& $(0.03)$ & $(0.08)$ & $(0.05)$ \\
7 & 0.03 & 0.08 & $0.25^{*}$ \\
& $(0.03)$ & $(0.05)$ & $(0.05)$ \\
8 & 0.06 & 0.06 & $0.32^{*}$ \\
\multirow{2}{*}{9} & $(0.04)$ & $(0.04)$ & $(0.04)$ \\
9 & $0.10^{*}$ & $0.15^{*}$ & $0.36^{*}$ \\
& $(0.04)$ & $(0.05)$ & $(0.04)$ \\
10 & $0.27^{*}$ & $0.15^{*}$ & $0.45^{*}$ \\
& $(0.07)$ & $(0.04)$ & $(0.04)$ \\
& & &
\end{tabular}

Table 4.1 - Out-of-sample monthly alphas of decile portfolios by ranking method, 1961-2010. At the end of each month $t$, alphas are estimated for each mutual fund using each method and the past 24 monthly returns. Funds are ranked, and equally-weighted decile portfolios are formed and held during month $t+2$. Alphas for these portfolios are estimated using OLS, with bootstrapped standard errors. 

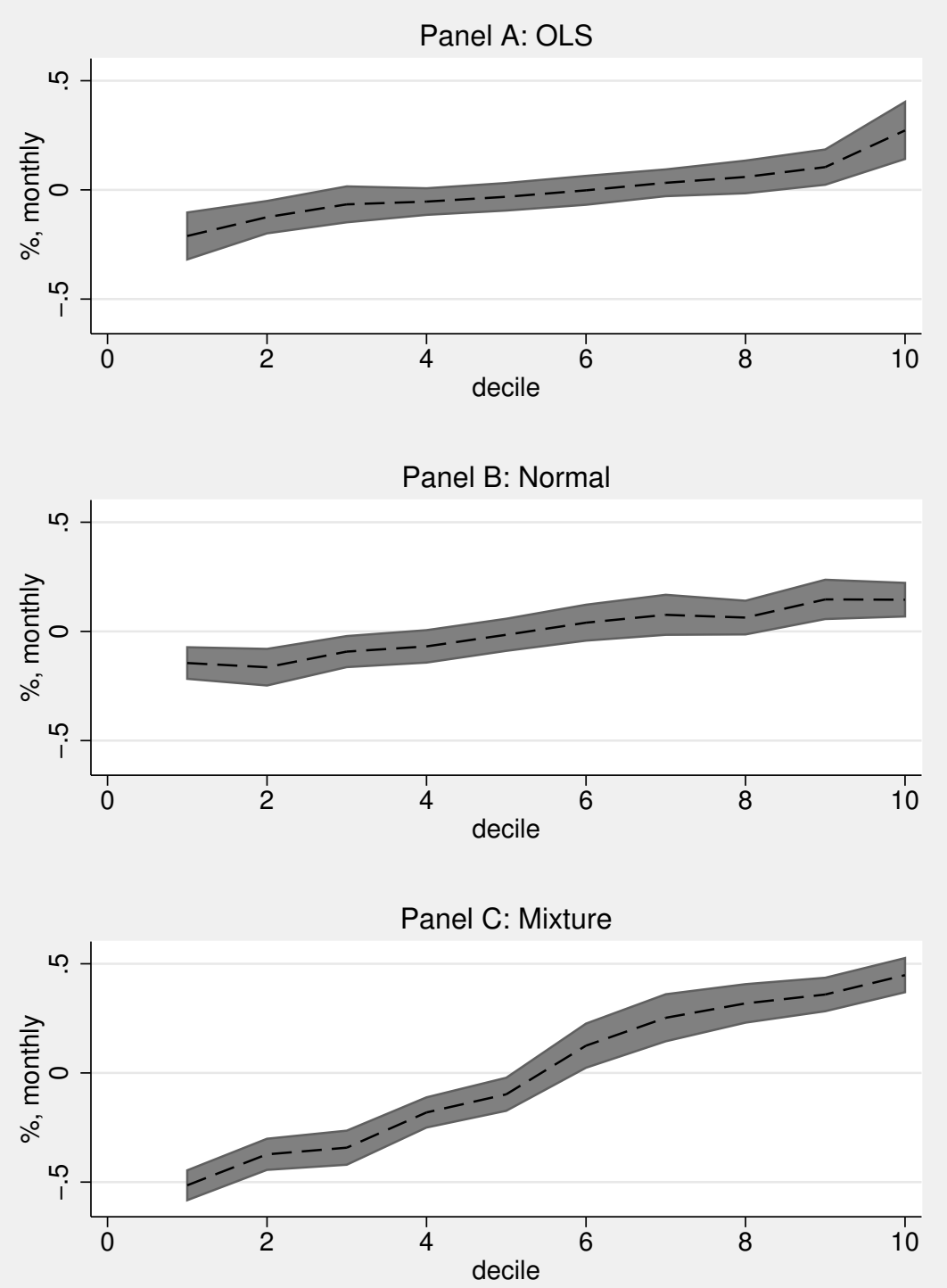

Figure 4.7 - Out-of-sample OLS alphas and 95\% confidence intervals for decile portfolios, by ranking method, 1961-2010. Data are taken from Table 4.1. Panel A shows decile portfolios formed from funds ranked on their OLS alphas. Panel B shows decile portfolios formed from funds ranked on their hierarchical Bayes alphas with a normal prior. Panel $\mathrm{C}$ shows decile portfolios formed from funds ranked on their hierarchical Bayes alphas with a mixture-of-normals prior. 


\subsection{Fund-by-fund alpha correlations}

The mixture model generates superior portfolios of funds; its fund-level estimates are better as well. Using the same two-year estimation windows, we can compare the estimated fund alphas for the interval $[t-23, t]$ to the estimated alphas for the non-overlapping interval $[t+2, t+25] .{ }^{31}$ Let the vector of estimated fund alphas generated by a particular model $k$ on the interval $\left[t_{a}, t_{b}\right]$ be denoted by $\widehat{\alpha}_{\left[t_{a}, t_{b}\right]}^{k}$, and define the correlation of the alphas on the first interval with the alphas on the second interval to be

$$
\rho_{t}^{k_{1}, k_{2}}=\text { correlation }\left(\widehat{\alpha}_{[t-23, t]}^{k_{1}}, \widehat{\alpha}_{[t+2, t+25]}^{k_{2}}\right) \text {. }
$$

Funds that do not appear in both intervals are dropped before the correlation is computed. I expect that the typical fund used in this part of the analysis is consequently better than average. Even if the level of alpha is biased upwards, this does not imply that the serial correlation is biased.

When comparing the performance of two models, $k_{1}$ and $k_{2}$, in predicting the estimates generated by model $k_{3}$, it is also useful to define:

$$
k_{1}, k_{3} \Delta_{t}^{k_{2}, k_{3}}=\rho_{t}^{k_{1}, k_{3}}-\rho_{t}^{k_{2}, k_{3}}
$$

Results are shown in Table 4.2. ${ }^{32}$ From the first three columns in Panel A, we see that all alphas are positively serially correlated, consistent with the predictability suggested by the performance of the decile portfolios. The last two columns show the correlations of alphas generated by the hierarchical models in the first period with

\footnotetext{
${ }^{31}$ By evaluating the estimation methods in this way, I assume that the short-term predictability in fund alphas is not completely offset by any un-modeled longer-term negative correlation.

${ }^{32}$ The same analysis was also performed using rank correlations with essentially identical results.
} 


\begin{tabular}{ccccc}
\hline \multicolumn{5}{c}{ Panel A: Serial Correlation of Estimated Fund Alphas, by Method } \\
\hline$\rho_{t}^{\text {OLS,OLS }}$ & $\rho_{t}^{\text {Normal,Normal }}$ & $\rho_{t}^{\text {Mixture,Mixture }}$ & $\rho_{t}^{\text {Normal,OLS }}$ & $\rho_{t}^{\text {Mixture }, \text { OLS }}$ \\
\hline $0.141^{*}$ & $0.116^{*}$ & $0.171^{*}$ & $0.104^{*}$ & $0.159^{*}$ \\
$(0.006)$ & $(0.005)$ & $(0.005)$ & $(0.004)$ & $(0.005)$ \\
\hline
\end{tabular}

\begin{tabular}{ccc}
\hline \multicolumn{2}{c}{ Panel B: Differences in Serial Correlations of Estimated Fund Alphas } \\
\hline Normal,Normal $\Delta_{t}^{\text {OLS,OLS }}$ & Mixture,Mixture $\Delta_{t}^{\text {OLS,OLS }}$ & Mixture,OLS $\Delta_{t}^{\text {OLS,OLS }}$ \\
\hline$-0.025^{*}$ & $0.031^{*}$ & $0.019^{*}$ \\
$(0.006)$ & $(0.004)$ & $(0.004)$ \\
\hline
\end{tabular}

Table 4.2 - In Panel A, serial correlations of estimated fund alphas are computed for rolling, non-overlapping, two-year intervals, 1961-2010. In the last two columns, the hierarchical models are used to predict OLS alphas. In Panel B, differences in these correlations are reported.

the OLS alphas from the second period. These are positive as well, and indicate that the positive serial correlations are not artifacts of the Bayesian procedure.

Panel B of Table 4.2 shows the differences in the correlations. In the second column, we can see that the mixture model's estimates are indeed more strongly serially correlated than the OLS estimates. Most critically, the third column shows that the mixture model predicts the OLS estimates better than OLS itself. 


\section{Section 5}

\section{Robustness Checks}

\subsection{Incubation}

One issue with the mutual fund data from CRSP is the incubation bias discussed in Evans (2010): "Mutual fund incubation is a strategy that some fund families use to develop new fund offerings. In incubation, families open multiple new funds, often with a limited amount of capital. At the end of an evaluation period, some funds are opened to the public, while the others are shut down before investors ever become aware of them... The Securities and Exchange Commission (SEC) allows fund families to advertise the incubation-period performance of these funds."

Thus, the apparent outperformance of young funds is largely the result of selection bias rather than managerial skill. To estimate the magnitude of the effect, Evans separates incubation-period returns from post-incubation-period returns by using the date when each fund's ticker was created. The creation of a ticker indicates that a fund will soon be sold to the public, and proxies for the end of incubation. When only post-incubation-period returns are considered, young funds no longer outperform older funds.

Ideally, this ticker creation date filter could be applied to the fund returns data before the rest of the analysis in this paper. Unfortunately I do not have ticker 
creation data from the National Association of Securities Dealers (NASD). As a cruder approximate approach, Evans also proposes an age filter that discards the first 36 months of each fund's returns. ${ }^{33}$ Although many valid data are lost, the data that remain are incubation bias free.

To check whether incubation is driving the results presented in this paper, I repeat the portfolio formation described in Section 4 using only funds with complete return histories during the window [ $t-59, t-24]$. That is, funds need 36 months of returns prior to the ranking period $[t-23, t]$.

OLS alphas for these portfolios are presented in Table 5.1, and graphically in Figure 5.1. There are no material differences between these alphas and those in the main analysis (Table 4.1 and Figure 4.7), indicating that incubation is not driving the main results of this paper.

\subsection{Alternative Alpha Distribution}

The finite mixture of normals defined in (2.2) was chosen because it is a simple, flexible, and familiar distribution that allows for both skewness and excess kurtosis while imposing neither. To check that the main results are not simply artifacts of this particular modeling choice, I re-estimate an alternative model that retains the standard return generating process in (2.1) but replaces the mixture of normals in (2.2) with the generalized unimodal distribution of Fernández and Steel (1998).

The true managerial alphas are now independent draws with density

$$
f\left(\alpha^{j}\right)=\frac{2}{\gamma+\frac{1}{\gamma}} \cdot \frac{1}{\sqrt{V}} \cdot\left[f_{\nu}\left(\frac{1}{\gamma} \cdot \frac{\alpha^{j}-\mu}{\sqrt{V}}\right) \cdot \mathbf{1}_{\alpha^{j} \geq \mu}+f_{\nu}\left(\gamma \cdot \frac{\alpha^{j}-\mu}{\sqrt{V}}\right) \cdot \mathbf{1}_{\alpha^{j}<\mu}\right],
$$

\footnotetext{
${ }^{33}$ See Table VIII in Evans (2010) for a complete comparison of the ticker creation date filter and the cruder age filter.
} 


\begin{tabular}{cccc}
\hline \multicolumn{5}{c}{ Out-of-Sample Monthly Alphas of Decile } \\
Portfolios After Age Filter \\
\hline Decile & OLS & Normal Prior & Mixture Prior \\
\hline 1 & $-0.22^{*}$ & $-0.16^{*}$ & $-0.49^{*}$ \\
& $(0.06)$ & $(0.04)$ & $(0.04)$ \\
2 & $-0.11^{*}$ & $-0.17^{*}$ & $-0.38^{*}$ \\
& $(0.04)$ & $(0.04)$ & $(0.04)$ \\
3 & -0.06 & -0.08 & $-0.31^{*}$ \\
& $(0.05)$ & $(0.06)$ & $(0.04)$ \\
4 & -0.05 & $-0.08^{*}$ & $-0.20^{*}$ \\
& $(0.03)$ & $(0.04)$ & $(0.04)$ \\
5 & -0.05 & -0.01 & $-0.12^{*}$ \\
& $(0.03)$ & $(0.04)$ & $(0.04)$ \\
6 & -0.01 & 0.00 & 0.06 \\
& $(0.04)$ & $(0.04)$ & $(0.05)$ \\
7 & 0.02 & 0.06 & $0.25^{*}$ \\
& $(0.03)$ & $(0.05)$ & $(0.07)$ \\
8 & 0.05 & 0.06 & $0.30^{*}$ \\
& $(0.04)$ & $(0.05)$ & $(0.05)$ \\
9 & $0.11^{*}$ & $0.10^{*}$ & $0.31^{*}$ \\
& $(0.05)$ & $(0.05)$ & $(0.03)$ \\
10 & $0.23^{*}$ & $0.16^{*}$ & $0.45^{*}$ \\
& $(0.07)$ & $(0.04)$ & $(0.04)$ \\
\hline \multirow{2}{*}{3} & & &
\end{tabular}

Table 5.1 - Out-of-sample monthly alphas of decile portfolios, after age filter, by ranking method, 1967-2010. At the end of each month $t$, alphas are estimated for each mutual fund using each method and the past 24 monthly returns - only for funds with a complete return history during the window $[\mathrm{t}-59, \mathrm{t}]$. Funds are ranked, and equally-weighted decile portfolios are formed and held during month $t+2$. Alphas for these portfolios are estimated using OLS, with bootstrapped standard errors. There is virtually no difference between the estimates here and those from the main analysis presented in Table 4.1, indicating that the main results are not attributable to the incubation bias of Evans (2010). 


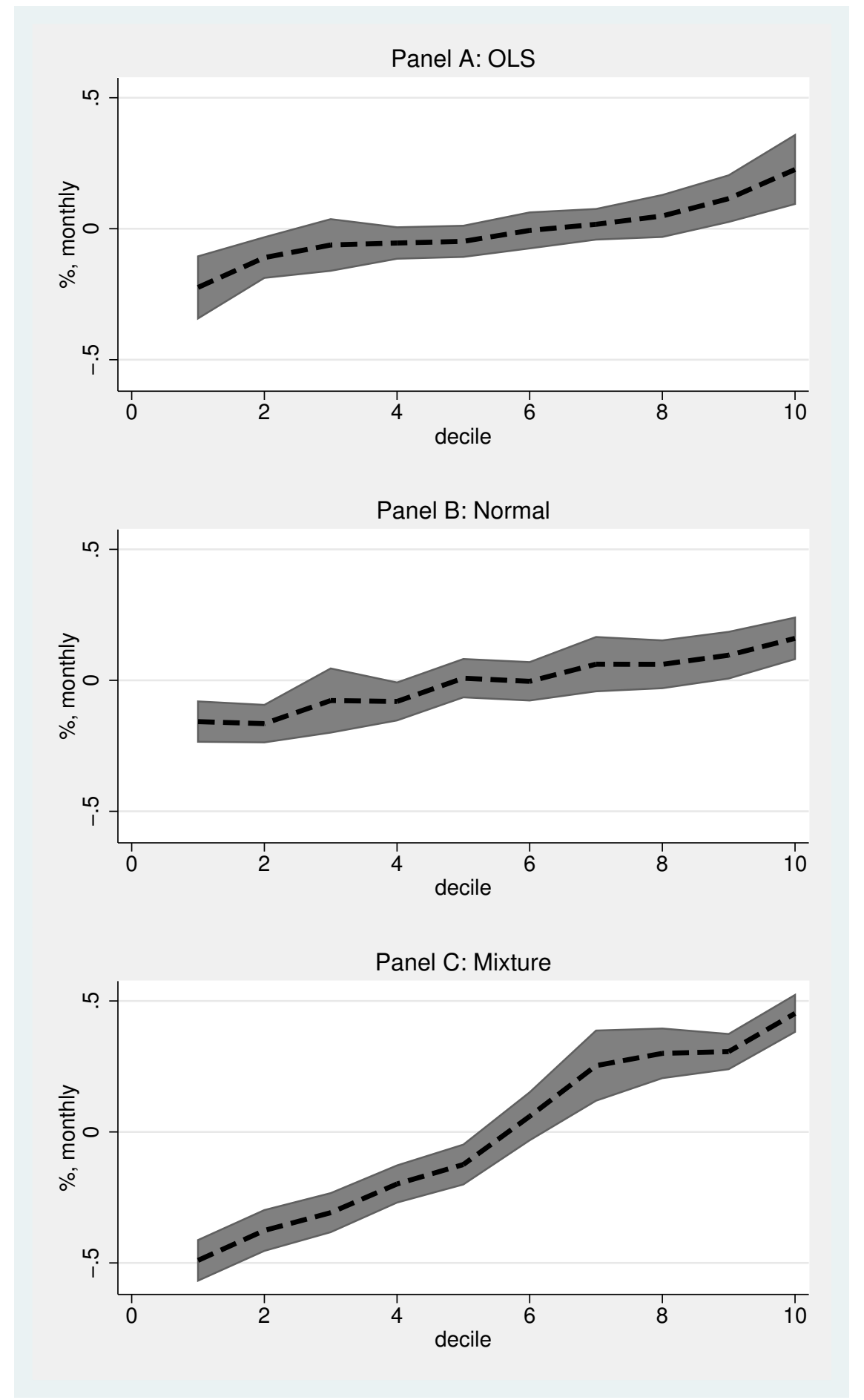

Figure 5.1 - Out-of-sample OLS alphas and 95\% confidence intervals for decile portfolios, after applying age filter, by ranking method, 1967-2010. Data are taken from Table 5.1. There are no significant differences in performance between these portfolios, formed using the age filter suggested in Evans (2010), and the original portfolios shown in Figure 4.7, indicating that incubation bias does not drive the results in this paper. 
where $f_{\nu}(x)$ is the density of the Student's $t$-distribution,

$$
f_{\nu}(x)=\frac{\left(\frac{\nu}{x^{2}+\nu}\right)^{\frac{\nu+1}{2}}}{\sqrt{\nu}} \cdot \frac{\Gamma\left(\frac{\nu+1}{2}\right)}{\Gamma\left(\frac{\nu}{2}\right) \Gamma\left(\frac{1}{2}\right)}
$$

and $\gamma>0$ is a skewness parameter. Values of $\gamma$ greater than one result in positive skewness, while values less than one result in negative skewness. Location parameter $\mu$ and scale parameter $\sqrt{V}$ have their usual interpretations. This distribution has only four parameters, one fewer than (2.2), while allowing skewness and kurtosis to vary independently. However, the distribution is unimodal by construction. Note than when $\gamma=1,(5.1)$ simplifies to the Student's $t$-distribution, which in turn nests the normal distribution.

Figure 5.2 depicts the influence of the skewness parameter $\gamma$. Setting $\gamma=1$ results in a symmetric density, while increasing $\gamma$ increases skewness. The mode of the distribution is fixed at $\mu$. Figure 5.3 shows the relationship between positively and negatively skewed densities. Holding other parameters fixed, setting $\gamma^{\prime}=\frac{1}{\gamma}$ reflects the original density with respect to $\mu$. Appendix B provides analytical expressions for the moments of this distribution. For details on the MCMC sampling algorithm, please refer to Fernández and Steel (1998).

Estimating alphas using this distribution rather than the finite mixture of normals and forming decile portfolios as in Section 4 produces the results shown in Table 5.2 and depicted graphically in Figure 5.4. For ease of comparison, the earlier results (using the mixture of normals prior on alpha) are reproduced. Although different in minor details, the portfolios formed using the alternative alpha distribution perform very similarly to the portfolios formed using the mixture prior on alpha. The ability of the hierarchical model to accurately measure - and predict - alphas does not depend on the particular distribution used to model alpha. 


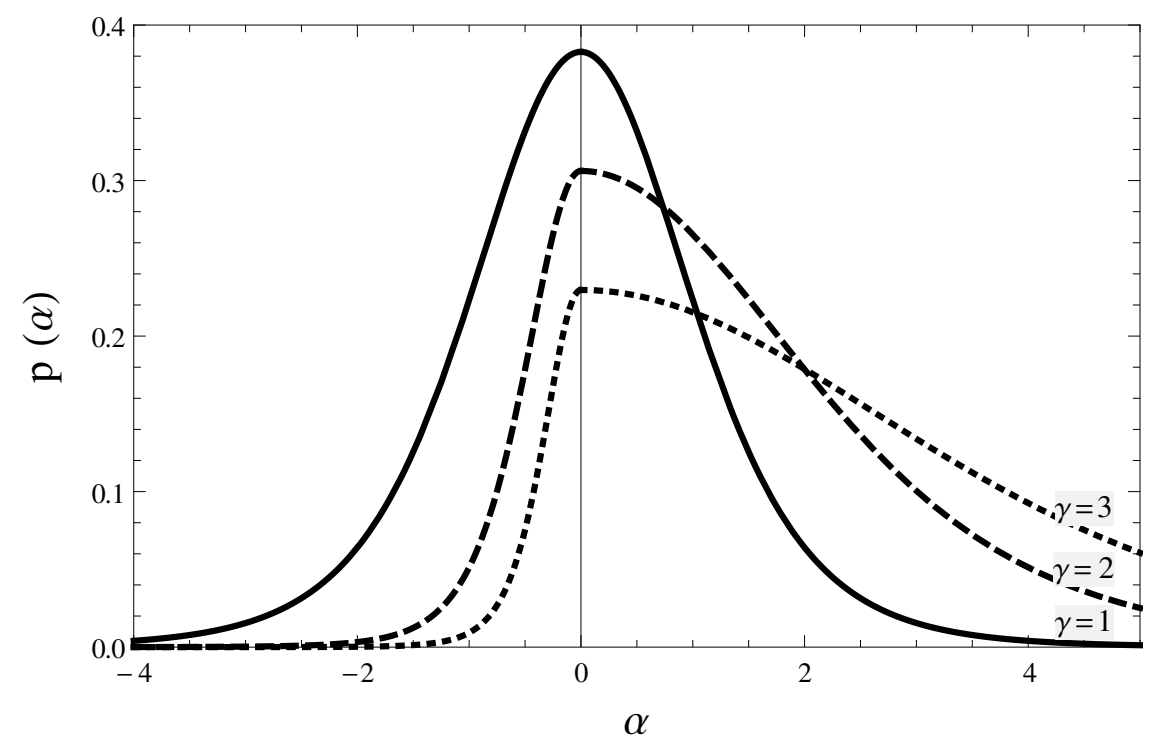

Figure 5.2 - Family of curves generated by (5.1), showing symmetric Student's $t$-distribution $(\gamma=1)$ and two positively skewed counterparts $(\gamma=2$, $\gamma=3)$. In all cases, $\mu=0, V=1$, and $\nu=6$.

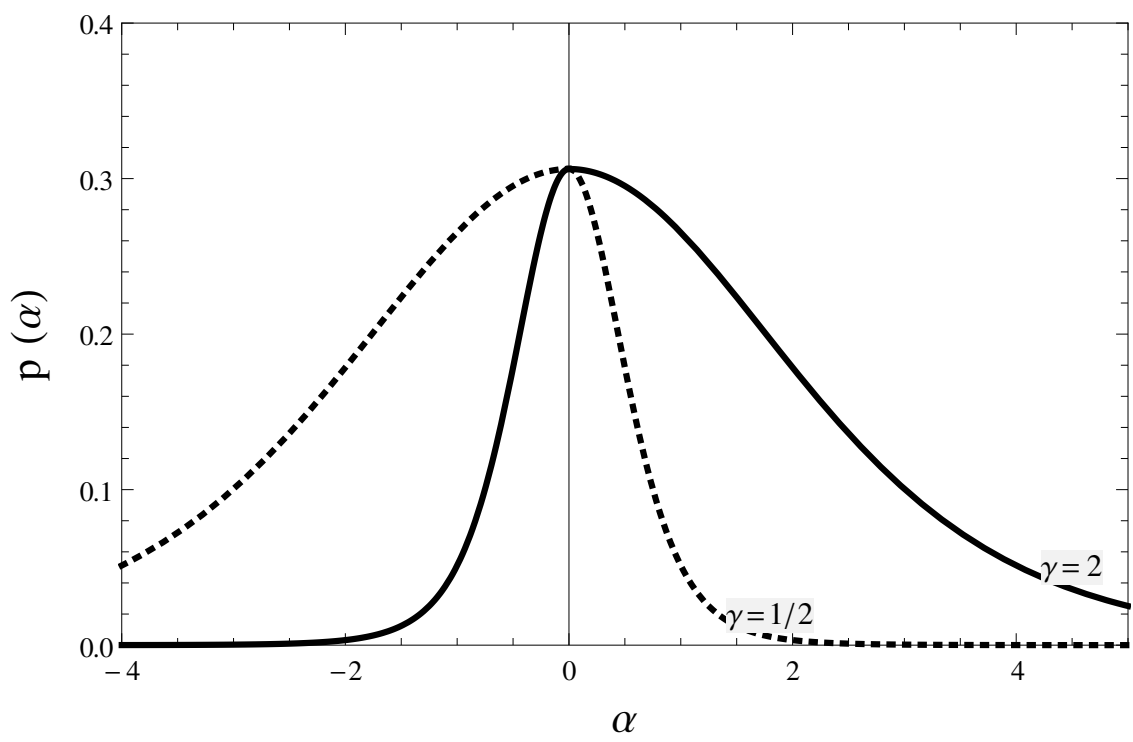

Figure 5.3 - Pair of curves generated by (5.1), showing positively skewed distribution $(\gamma=2)$ and its negatively skewed mirror image $\left(\gamma=\frac{1}{2}\right)$. In both cases, $\mu=0, V=1$, and $\nu=6$. In general, $p(\alpha-\mu \mid \mu, V, \gamma, \nu)=$ $p\left(-(\alpha-\mu) \mid \mu, V, \frac{1}{\gamma}, \nu\right)$, i.e. taking the reciprocal of $\gamma$ reflects the density with respect to $\mu$. 


\begin{tabular}{ccc}
\hline \multicolumn{3}{c}{$\begin{array}{c}\text { Out-of-Sample Monthly Alphas } \\
\text { of Decile Portfolios }\end{array}$} \\
\hline Decile & Generalized Unimodal Prior & Mixture Prior \\
\hline 1 & $-0.49^{*}$ & $-0.51^{*}$ \\
& $(0.07)$ & $(0.03)$ \\
2 & $-0.34^{*}$ & $-0.37^{*}$ \\
3 & $(0.06)$ & $(0.04)$ \\
& $-0.26^{*}$ & $-0.34^{*}$ \\
4 & $(0.06)$ & $(0.04)$ \\
& -0.06 & $-0.18^{*}$ \\
5 & $(0.07)$ & $(0.04)$ \\
& -0.06 & $-0.10^{*}$ \\
6 & $(0.06)$ & $(0.04)$ \\
& $0.21^{*}$ & $0.25^{*}$ \\
7 & $(0.08)$ & $(0.05)$ \\
& $0.21^{*}$ & $0.25^{*}$ \\
8 & $(0.06)$ & $(0.05)$ \\
& $0.33^{*}$ & $0.32^{*}$ \\
9 & $(0.06)$ & $(0.04)$ \\
& $0.27^{*}$ & $0.36^{*}$ \\
& $(0.07)$ & $(0.04)$ \\
& $0.50^{*}$ & $0.45^{*}$ \\
& $(0.06)$ & $(0.04)$ \\
& &
\end{tabular}

Table 5.2 - Out-of-sample monthly alphas of decile portfolios formed using alternative alpha distribution, 1963-2010. At the end of each month $t$, alphas are estimated for each mutual fund using each method and the past 24 monthly returns. Funds are ranked, and equally-weighted decile portfolios are formed and held during month $t+2$. Alphas for these portfolios are estimated using OLS, with bootstrapped standard errors. Values for the mixture prior are taken from Table 4.1. There are no material differences between these two sets of estimates, indicating that the main results are not sensitive to the particular distribution used to model the cross-section of alpha. 


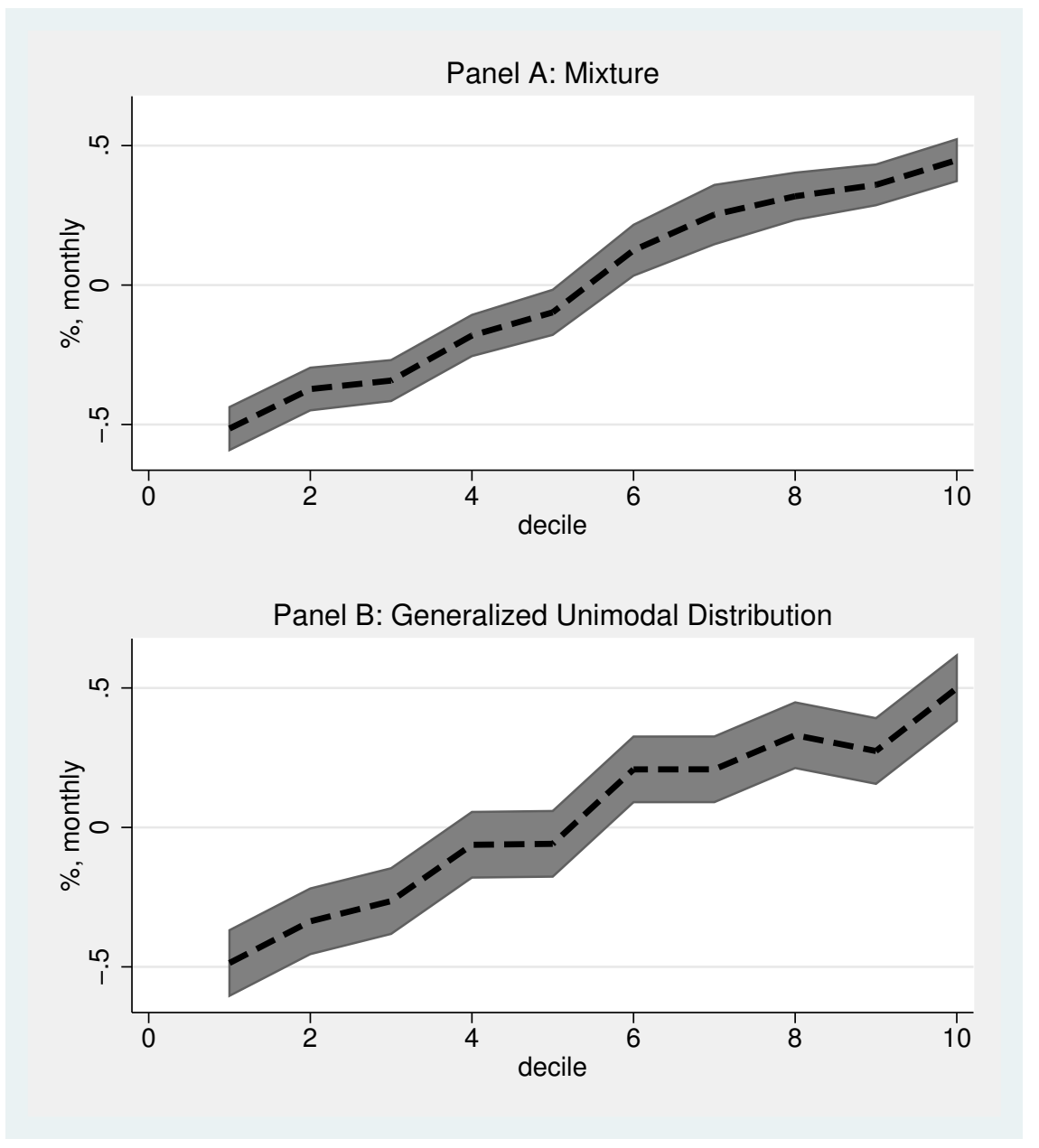

Figure 5.4 - Out-of-sample monthly alphas of decile portfolios formed using alternative alpha distribution, 1963-2010. Data are taken from Table 5.2. Although different in minor details, the portfolios formed using the alternative alpha distribution perform very similarly to the portfolios formed using the mixture prior on alpha. The ability of the hierarchical model to accurately measure - and predict - alphas does not depend on the particular distribution used to model alpha. 


\subsection{Non-normal Idiosyncratic Errors}

One assumption embedded in (2.1) is that the idiosyncratic errors in fund returns are normally distributed. It is worthwhile to examine the validity of this assumption. Although the out-of-sample portfolio performance in Section 4.4 is robust to any misspecification of the error distribution, the estimated moments of the cross-sectional distribution of alpha, shown in Figure 4.3, are influenced by modeling choices with respect to error distributions. ${ }^{34}$

If true idiosyncratic errors are skewed, then the residuals from (2.1) will also be skewed. The main estimations from Section 4 imposed only the condition of zero expectation on the errors. Residuals are computed using the Bayesian mixture parameter estimates,

$$
e_{t}^{j}=\left(R_{t}^{j}-R_{t}^{f}\right)-\widehat{\alpha^{j}}-F^{t} \widehat{\beta^{j}}
$$

and the skewness of each fund's residuals are computed for each 24-month interval during the sample,

$$
\operatorname{skew}\left(e_{[t-23, t]}^{j}\right)=\frac{\frac{1}{24} \sum_{s=t-23}^{t}\left(e_{s}^{j}\right)^{3}}{\left(\frac{1}{24} \sum_{s=t-23}^{t}\left(e_{s}^{j}\right)^{2}\right)^{\frac{3}{2}}} .
$$

For each month $t$, we can examine the distribution of $\operatorname{skew}\left(e_{[t-23, t]}^{j}\right)$ across all funds $j$. If true errors are normal, we would expect this distribution to be very concentrated near zero. In a finite sample, we will always observe non-zero residual skewness, regardless of the true distribution. Our principal concern, however, is whether the pronounced positive skewness in the cross-sectional distribution of alpha, as depicted

\footnotetext{
${ }^{34}$ Section 4 presented findings of skewed and fat-tailed alphas, conditional on normally distributed errors. If the errors themselves are skewed and fat-tailed, while true alphas are not, the estimated alphas may still exhibit the skewness and excess kurtosis shown in Figure 4.3 - spuriously inheriting their distribution from the true errors.
} 
in Panel C of Figure 4.3, could have been inherited from a positively skewed error distribution.

Figure 5.5 shows the distribution of $\operatorname{skew}\left(e_{[t-23, t]}^{j}\right)$ for nine consecutive, nonoverlapping 24-month intervals. In all cases, there is no indication that residuals tend to be positively skewed. If anything, some of the histograms show a tendency towards negative skewness. These diagnostic plots suggest that the (strong) assumption of normal idiosyncratic errors is not driving the cross-sectional estimates in Figure 4.3, and strengthen the evidence for positive skewness of true managerial alphas.

To completely account for possibly non-normal idiosyncratic errors, the model in (2.1) could be expanded. Instead of assuming that errors are normal, we could instead assume that they are drawn from the generalized unimodal distribution of Fernández and Steel (1998), described in Section 5.2. ${ }^{35}$

\subsection{Alternative Ranking Rules}

In the main analysis, funds are ranked according to their alpha point estimates. As noted by Mamaysky et al. (2007), this approach to fund sorting may populate the extreme decile portfolios with funds with the most imprecisely estimated alphas, rather than with the truly best and worst funds. Kosowski et al. (2006) and Fama and French (2010) propose sorting on the $t$-statistics of the estimated alphas, rather than the alphas themselves, to exploit the superior statistical properties of the $t$-statistic.

To check that the decile portfolios formed using OLS estimates are not being unfairly handicapped by the choice of any specific ranking rule, I repeat the main analysis after sorting on funds' $t$-statistics. For the Bayesian hierarchical estimates

\footnotetext{
${ }^{35}$ Care would need to be taken to correctly impose the zero-mean condition, however, since the parameter $\mu$ in their distribution represents the mode, not the mean. Moreover, some valid combinations of the distribution parameters $(\mu, V, \gamma, \nu)$ lead to distributions with infinite means. See Appendix B.
} 

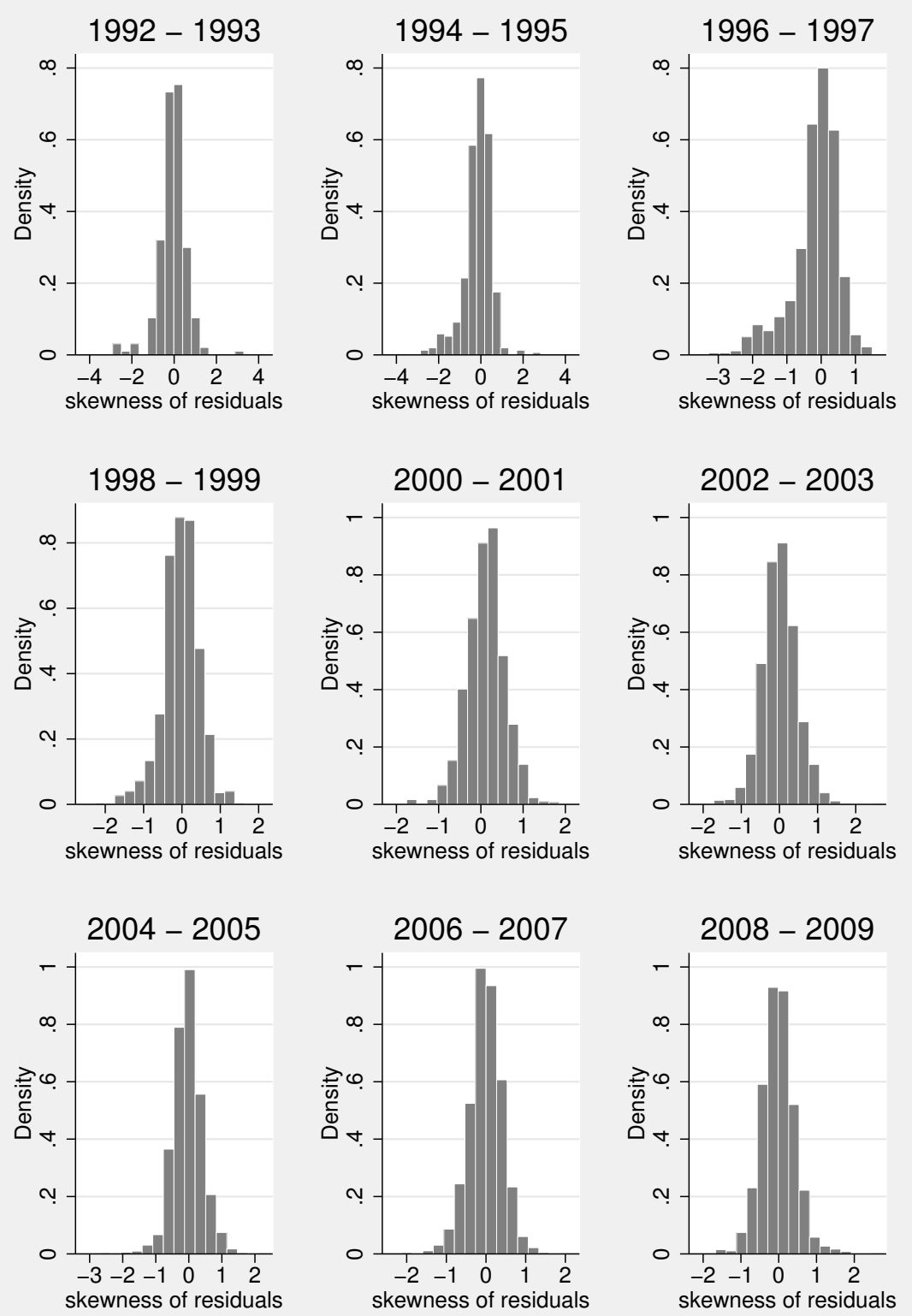

Figure 5.5 - Histograms of fund-level residual skewness, computed according to (5.3) and (5.4), for nine non-overlapping 24-month intervals, 19922009. There is no evidence that residuals are generally positively skewed. Thus, the positive skewness of alphas in Figure 4.3 cannot be attributed to any un-modeled non-normality of idiosyncratic errors. 
using both normal and mixture priors, I rank according to the pseudo-t-statistic $\frac{\sqrt{T} \cdot \widehat{\alpha^{j}}}{S D\left(\widehat{\alpha^{j}}\right)}$, where $T$ is the number of return observations for fund $j$.

As before, ranked funds are grouped into equally-weighted decile portfolios, and these portfolios are rebalanced monthly. Alphas for these portfolios are estimated using OLS. The results are reported in Table 5.3. For comparison, the main results with portfolios formed by ranking funds on $\widehat{\alpha^{j}}$ are showin in Table 4.1.

The change in sort variable did not materially affect the Bayesian portfolios. Posterior distributions of $\widehat{\alpha^{j}}$ automatically account for noisy data by placing relatively more emphasis on the prior-distributions with means of zero in this case. ${ }^{36}$ If the data for a particular fund $j$ are especially noisy, $\widehat{\alpha^{j}}$ will tend towards zero as well, i.e. it will be more likely to be assigned to deciles 5 or 6 , not deciles 1 or 10 .

The OLS portfolios also performed almost identically, despite the change in sort variable. Although $t$-statistics are, in general, better behaved than simple point estimates, it is clear that the main results of presented here do not depend on the choice of sort variable.

\subsection{Alternative Performance Measures}

\subsubsection{Decile Portfolio Performance}

To check that the results are not linked to a specific performance measure - the fourfactor alpha used in the main analysis - I compute mean excess returns and Sharpe ratios for the decile portfolios. Even though the funds are still ranked according to $\widehat{\alpha^{j}}$ and grouped into decile portfolios accordingly, these portfolios are now evaluated using two simple alternative performance measures.

\footnotetext{
${ }^{36}$ See Section A.3.
} 


\begin{tabular}{cccc}
\hline \multicolumn{4}{c}{$\begin{array}{c}\text { Out-of-Sample Monthly Alphas of Decile } \\
\text { Portfolios Sorted on }\end{array}$-Statistics } \\
\hline Decile & OLS & Normal Prior & Mixture Prior \\
\hline 1 & $-0.22^{*}$ & $-0.19^{*}$ & $-0.51^{*}$ \\
& $(0.06)$ & $(0.04)$ & $(0.04)$ \\
2 & $-0.11^{*}$ & $-0.10^{*}$ & $-0.37^{*}$ \\
& $(0.04)$ & $(0.04)$ & $(0.04)$ \\
3 & -0.06 & $-0.11^{*}$ & $-0.34^{*}$ \\
& $(0.04)$ & $(0.04)$ & $(0.04)$ \\
4 & -0.05 & -0.02 & $-0.18^{*}$ \\
& $(0.03)$ & $(0.04)$ & $(0.04)$ \\
5 & -0.02 & -0.03 & $-0.10^{*}$ \\
& $(0.04)$ & $(0.04)$ & $(0.04)$ \\
6 & 0.01 & 0.02 & $0.12^{*}$ \\
& $(0.03)$ & $(0.04)$ & $(0.05)$ \\
7 & 0.00 & 0.08 & $0.25^{*}$ \\
& $(0.03)$ & $(0.05)$ & $(0.05)$ \\
8 & $0.09^{*}$ & 0.06 & $0.32^{*}$ \\
& $(0.04)$ & $(0.04)$ & $(0.04)$ \\
9 & $0.08^{*}$ & $0.14^{*}$ & $0.36^{*}$ \\
& $(0.04)$ & $(0.04)$ & $(0.04)$ \\
& $0.26^{*}$ & $0.14^{*}$ & $0.45^{*}$ \\
& $(0.06)$ & $(0.04)$ & $(0.04)$ \\
& & &
\end{tabular}

Table 5.3 - Out-of-sample monthly alphas of decile portfolios sorted on t-statistics, by ranking method, 1963-2010. At the end of each month $t$, alphas are estimated for each mutual fund using each method and the past 24 monthly returns - only for funds with a complete return history during the window $[\mathrm{t}-59, \mathrm{t}]$. Funds are ranked according to the t-statistics of their estimated alphas - as opposed to the alpha point estimates - and equallyweighted decile portfolios are formed and held during month $t+2$. Alphas for these portfolios are estimated using OLS, with bootstrapped standard errors. There is virtually no difference between the estimates here and those from the main analysis in Table 4.1. 
Mean excess portfolio returns are shown in Table 5.4. Excess returns are calculated with respect to the risk-free rate. Portfolios formed using the Bayesian mixture $\widehat{\alpha^{j}}$ exhibit a mean excess return that is strictly increasing in decile order, with a difference (decile 10 - decile 1 ) of $1.23 \%$ per month. Portfolios formed using OLS $\widehat{\alpha^{j}}$ also produce monotonically increasing mean excess returns, although the difference between extreme deciles is only $0.47 \%$ per month.

Portfolio Sharpe ratios are shown in Table 5.5. Again, performance is strictly increasing in decile rank for both Bayesian mixture and OLS portfolios. But the difference between extreme deciles (decile 10 - decile 1 ) is 0.87 for the mixture prior, while it is only 0.29 for OLS.

The results using these two alternative performance metrics is entirely consistent with the main results using four-factor alphas. I can't rule out the possibility that using additional and/or different factors might erode or even reverse the results. Nevertheless, the strength of these results suggests that the main results in Section 4 are robust to reasonable alternative performance measures.

\subsubsection{Fund-Level Return Predictability}

In addition to evaluating decile portfolio performance, we can also consider alternative measures of fund-level predictability. Section 4.5 showed that the estimated alphas were predictable across non-overlapping 2-year periods. We can also evaluate fund return predictability.

Using monthly data as in Section 4, estimate fund alphas and factor loadings for each month $t$ and use these to compute predicted excess fund returns, $\tilde{R}$, during month $t+2$,

$$
\tilde{R}_{t+2}^{j}=\hat{\alpha}_{t}^{j}+F_{t+2} \hat{\beta}_{t}^{j}
$$




\begin{tabular}{cccc}
\hline \multicolumn{4}{c}{ Mean Excess } \\
Decile Portfolios, in percentage terms \\
\hline Decile & OLS & Normal Prior & Mixture Prior \\
\hline 1 & 0.28 & 0.28 & -0.12 \\
& $(0.21)$ & $(0.19)$ & $(0.20)$ \\
2 & 0.35 & 0.28 & 0.13 \\
& $(0.20)$ & $(0.19)$ & $(0.19)$ \\
3 & 0.36 & 0.36 & 0.15 \\
& $(0.19)$ & $(0.19)$ & $(0.19)$ \\
4 & 0.37 & 0.38 & 0.31 \\
& $(0.19)$ & $(0.20)$ & $(0.19)$ \\
5 & $0.39^{*}$ & $0.44^{*}$ & 0.35 \\
& $(0.19)$ & $(0.20)$ & $(0.20)$ \\
6 & $0.42^{*}$ & $0.49^{*}$ & $0.50^{*}$ \\
& $(0.19)$ & $(0.20)$ & $(0.20)$ \\
7 & $0.46^{*}$ & $0.52^{*}$ & $0.55^{*}$ \\
& $(0.19)$ & $(0.20)$ & $(0.20)$ \\
8 & $0.49^{*}$ & $0.51^{*}$ & $0.68^{*}$ \\
& $(0.19)$ & $(0.20)$ & $(0.20)$ \\
9 & $0.56^{*}$ & $0.61^{*}$ & $0.77^{*}$ \\
& $(0.21)$ & $(0.20)$ & $(0.20)$ \\
10 & $0.75^{*}$ & $0.55^{*}$ & $1.11^{*}$ \\
& $(0.23)$ & $(0.19)$ & $(0.21)$ \\
& & &
\end{tabular}

Table 5.4 - Mean excess returns of decile portfolios, by ranking method, 1963-2010. Returns are in excess of the risk-free rate. For comparison, the mean monthly return of the Fama-French market factor, mktrf, was $0.40 \%$ over the same period. 


\begin{tabular}{cccc}
\hline \multicolumn{4}{c}{$\begin{array}{c}\text { Annualized Sharpe Ratios of } \\
\text { Decile Portfolios }\end{array}$} \\
\hline Decile & OLS & Normal Prior & Mixture Prior \\
\hline 1 & 0.19 & 0.22 & -0.09 \\
2 & 0.26 & 0.21 & 0.10 \\
3 & 0.28 & 0.27 & 0.11 \\
4 & 0.29 & 0.28 & 0.24 \\
5 & 0.31 & 0.32 & 0.26 \\
6 & 0.32 & 0.36 & 0.37 \\
7 & 0.35 & 0.38 & 0.41 \\
8 & 0.37 & 0.37 & 0.49 \\
9 & 0.40 & 0.44 & 0.55 \\
10 & 0.48 & 0.42 & 0.78 \\
\hline
\end{tabular}

Table 5.5 - Annualized Sharpe ratios of decile portfolios, by ranking method, 1963-2010. Returns are in excess of the risk-free rate. For comparison, the annualized Sharpe ratio of the Fama-French market factor, mktrf, was 0.30 over the same period.

under the assumption of constant factor loadings. For each month $t$, compute the correlation of predicted returns, $\tilde{R}_{t}^{j}$, and realized returns, $R_{t}^{j}$, across all funds $j$,

$$
\rho_{t}^{k}=\operatorname{correlation}\left(\tilde{R}_{t}^{j}, R_{t}^{j}\right)
$$

where $k$ indicates how the fund parameters were estimated; either OLS or the mixture model. Finally, compute the correlation gap, $\delta_{t}$ - the difference between the two correlation time series:

$$
\delta_{t}=\rho_{t}^{\text {mixture }}-\rho_{t}^{O L S} .
$$

Since higher correlations indicate stronger predictability, positive $\delta_{t}$ indicates that the mixture model predicts returns better than does OLS. 


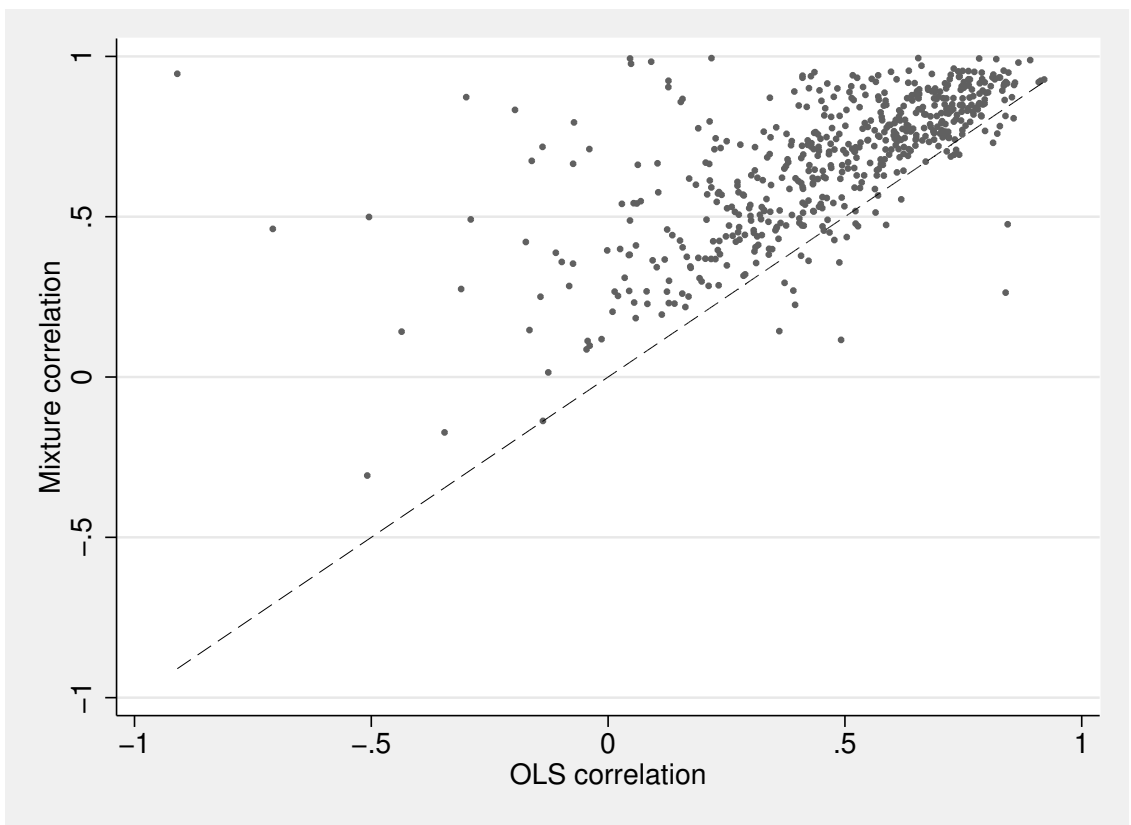

Figure 5.6 - Scatterplot showing monthly $\rho_{t}^{\text {mixture }}$ versus $\rho_{t}^{O L S}, 1963-2010$. The $45^{\circ}$ line is shown for reference. Nearly all the data points lie above the line, indicating systematically higher correlations between realized returns and Bayesian mixture predicted returns, than between realized returns and OLS predicted returns.

Figure 5.6 shows a scatterplot of $\rho_{t}^{\text {mixture }}$ versus $\rho_{t}^{O L S}$ for the period 1963-2010. Each data point represents one month of data. Across the entire time sample, nearly all of the data lie above the reference $45^{\circ}$ line: the Bayesian mixture estimates predict returns much better than do the OLS estimates. This result is robust to any potential mis-specification of the return generating process in (2.1), as we are predicting observed returns, not estimated alphas.

Figure 5.7 illustrates the distribution of the correlation gap, $\delta_{t}$, as defined in (5.7). Panel A shows a histogram of the correlation gap. The mean of the distribution is clearly positive, and almost none of the mass lies below zero. In almost every period, the Bayesian mixture estimates outperform the OLS estimates. Panel B shows the same series graphed as a function of time. The outperformance, as evidenced by positive $\delta_{t}$, is a consistent feature across the entire time sample. 

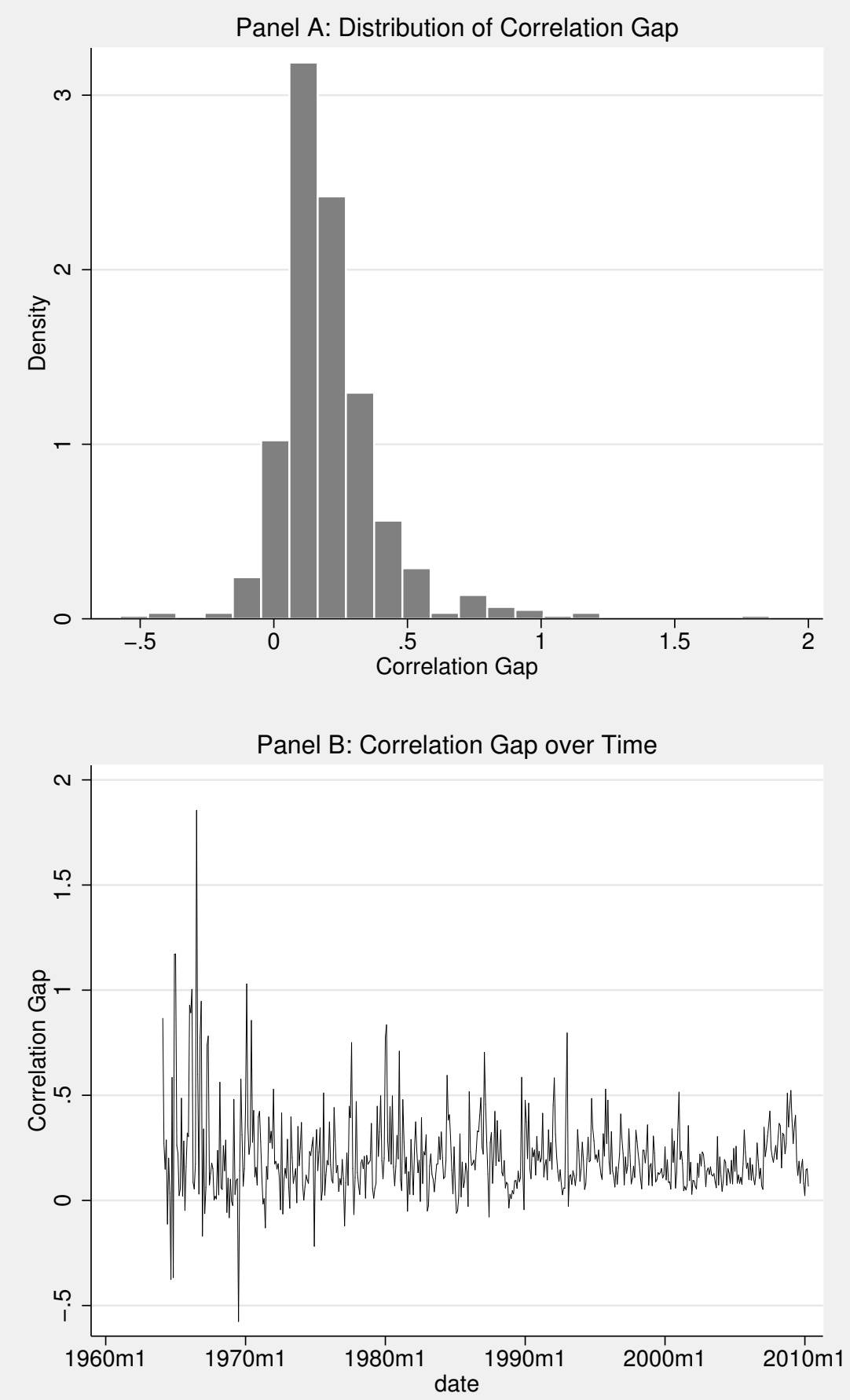

Figure 5.7 - Distribution of correlation gap, $\delta_{t}$, as defined in (5.7), 19632010. Panel A shows a histogram of the monthly observations, clearly indicating a positive mean and very little mass below zero. Panel B shows $\delta_{t}$ over time, illustrating that the Bayesian mixture estimates outperform OLS estimates across the entire time sample. 


\section{Part III}

\section{Discussion}




\section{Section 6}

\section{Implications}

The persistent performance of the decile portfolios is impossible to fully reconcile with the rational model of Berk and Green (2004). In their model, managers can possess investing skill but do not exhibit persistent non-zero alphas due to convexities in the costs of managing their funds. As funds grow in size, positive alpha becomes increasingly difficult to generate. In equilibrium all funds have zero alphas in expectation, but vary in size according to their managers' varying levels of skill. This equilibrium requires investors to evaluate managers in the context of a known crosssectional distribution of alpha, and to dynamically re-allocate capital across funds in each period.

In contrast to the equilibrium predictions of Berk and Green, I find markedly persistent performance in the short-term. At least one of their assumptions must not hold. Costs may not be convex. Reuter and Zitzewitz (2010) use fund flows associated with discrete changes in Morningstar fund ratings to show that their effect on fund performance is inconsistent with the Berk and Green hypothesis. There may be frictions associated with capital re-allocation that prevent investors from responding immediately to new estimates of manager skill.

The most indisputable deviation from the Berk and Green world, however, is that investors do not know the true cross-sectional distribution of alpha. In reality, as 
in the model presented here, this distribution and the individual fund alphas must be estimated simultaneously. Such an analysis requires MCMC methods-somewhat beyond the capabilities of the typical mutual fund investor, or even the typical financial advisor. The sheer difficulty of the evaluation is sufficient to cause slow learning, even among competent and vigilant investors. Even if all of the other assumptions of Berk and Green hold, this slow learning implies the short-term persistence of alphas that I document.

Flow predictability regressions presented below show that investors do respond to the information contained in the Bayesian mixture alphas. Whatever heuristics they employ to evaluate funds must incorporate the intuition of my model if not its details.

\subsection{Convexity of fund flows}

Fund flows have a convex relationship with past fund performance. Outflows in response to poor performance are less pronounced than the inflows in response to good performance. ${ }^{37}$ Lynch and Musto (2003) offer an explanation: if poor performance makes changes in investment strategy more likely, then poor performance is less informative than good performance regarding future returns. Berk and Green (2004) also predict a convex flow-performance relationship - a consequence of the convex costs of management in their model. ${ }^{38}$

Another possibility is that the optimal updating rule for posterior skill estimates is itself convex. While a normal prior (along with a normal likelihood) gives rise to a linear updating rule, the mixture prior proposed here generates an updating rule that

${ }^{37}$ See, e.g., Sirri and Tufano (1998) and Chevalier and Ellison (1997).

${ }^{38}$ See Figure 3 in Berk and Green (2004). 
is nonlinear for observed returns that are not extremely far from zero, and convex over the region of greatest interest. ${ }^{39}$

Assume that the true cross-sectional distribution of skill is a mixture-of-normals, as in (2.2). For comparison, I also consider a normal prior on skill. To obtain the best approximation, the mean and variance of the normal prior are set to match the mean and variance of the mixture distribution:

$$
\mu=\left(1-w_{2}\right) \cdot \mu_{1}+w_{2} \cdot \mu_{2}
$$

and

$$
V=\left(1-w_{2}\right) \cdot V_{1}+w_{2} \cdot V_{2}+w_{2} \cdot\left(1-w_{2}\right) \cdot\left(\mu_{1}-\mu_{2}\right)^{2}
$$

Assume that fund returns are simply managerial skill plus Gaussian disturbances: a simplified version of the model in (2.1) with no risk factors.

After observing a single return $R$, investors update their estimates of managerial skill. The posterior expectation of skill under the normal prior is:

$$
E_{\text {normal }}[\alpha]=\left(\frac{\mu}{V}+\frac{R}{V_{\varepsilon}}\right) \cdot\left(\frac{1}{V}+\frac{1}{V_{\varepsilon}}\right)^{-1}
$$

The posterior expectation of skill under the mixture prior is:

$$
E_{\text {mixture }}[\alpha]=\sum_{i=1}^{N} \widetilde{w}_{i} \cdot\left(\frac{\mu_{i}}{V_{i}}+\frac{R}{V_{\varepsilon}}\right) \cdot\left(\frac{1}{V_{i}}+\frac{1}{V_{\varepsilon}}\right)^{-1}
$$

where

$$
\widetilde{w}_{i}=\frac{w_{i}}{\sqrt{V_{i}+V_{\varepsilon}}} \cdot \exp \left[-\frac{1}{2} \cdot \frac{\left(R-\mu_{i}\right)^{2}}{V_{i}+V_{\varepsilon}}\right]
$$

\footnotetext{
${ }^{39}$ The details depend on the model parameters, but the intuition is that extreme observations are almost certainly drawn from the mixture component with the highest variance. Since this component is normal, the updating rule has a linear asymptote.
} 


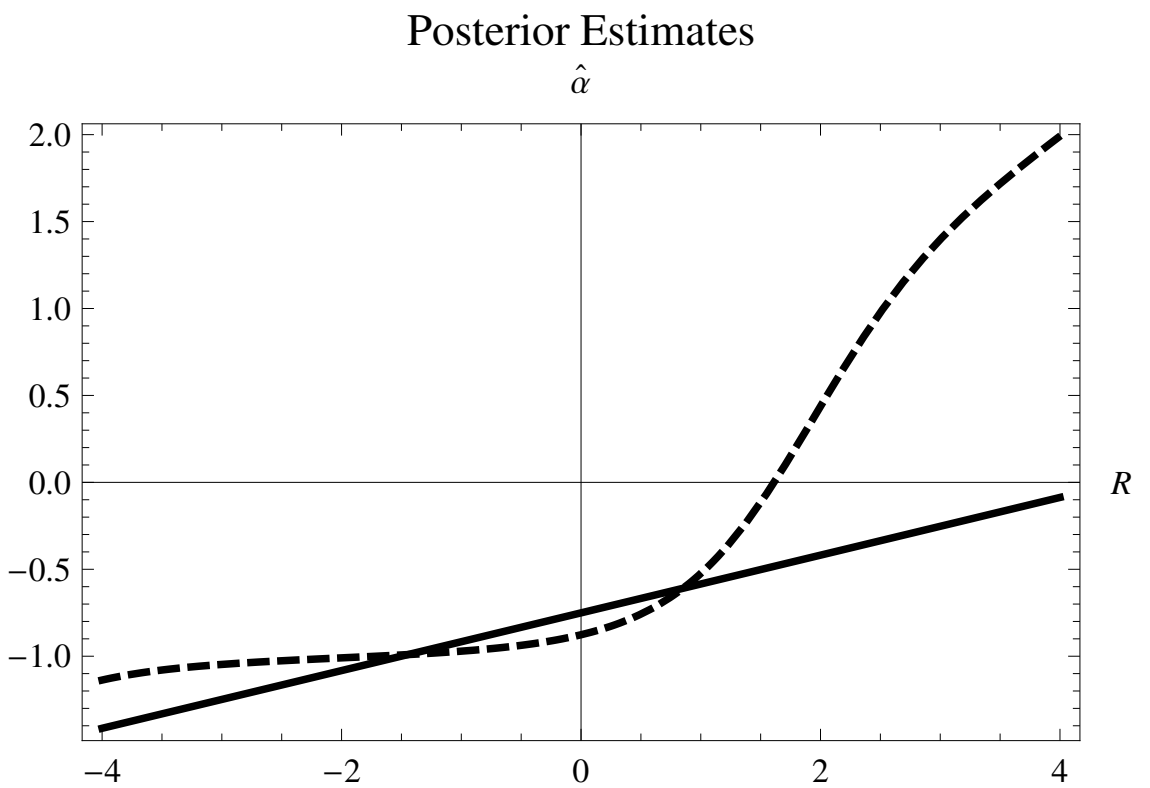

Figure 6.1 - Updating rules for managerial skill with a mixture-of-normals prior (dashed line) and a normal prior with matching first and second moments (solid line). $R$ is a monthly return measured in percent. The parameters of the mixture distribution are: $\mu_{1}=-1, \mu_{2}=0, V_{1}=0.01, V_{2}=1$, and $w_{2}=0.1$. Plugging these into (6.1) and (6.2), we get the following parameters for the "matching" normal distribution: $\mu=-0.9$ and $V=0.2$. The updating rule for the normal prior is linear. The updating rule for the mixture prior has a linear asymptote but is nonlinear for non-extreme returns, and is generally convex for monthly returns between -4 and +4 percent.

To make this more concrete, the posterior expectations, (6.3) and (6.4), of alpha corresponding to each of these priors after a single return $R$ are shown in Figure 6.1. Here we can easily see how sensitive the posterior beliefs are to the investor's prior, even when both possible priors have matching first and second moments. The updating rule for the mixture prior is generally convex for non-extreme monthly returns. If the relationship between skill estimates and observed returns is convex, and the relationship between fund flows and skill estimates is not too concave, we should expect to observe convex flows.

The mixture-of-normals prior is not special in this regard. Any non-normal prior will generate a nonlinear updating rule. A more flexible prior than the two-component 
mixture should be able to match the observed flow-performance relationship even more closely.

\subsection{Predictability of fund flows}

This paper describes a method of accurately estimating mutual fund alphas that fully exploits the information contained in the cross-section of funds. It is natural to ask what resemblance this bears to the methods investors actually use to evaluate funds. Although their methods are not observable, investors' conclusions are revealed by the fund flow data.

Results for a series of fund flow predictability regressions are shown in Tables 6.1 and 6.2. The dependent variable is the flow ratio, defined as the net fund flow divided by the fund's total assets under management in the previous month. Regression (1) is patterned after the baseline regression model of Sirri and Tufano (1998). This shows that investors are highly responsive to past returns, although they are more responsive to high returns than to low returns - the effect of being in the top quintile of past returns is positive and significant even after controlling for average past returns, $\overline{R_{t}-R_{m k t}}$. This convexity is a robust feature of mutual fund flows.

Regression (2) adds the lagged OLS alpha as a predictor, while regression (3) adds the lagged Bayesian mixture alpha. Regression (4) includes them both. Regression (2) shows that investors are indeed responsive to the information in OLS alphas. This information does not subsume the effect of past returns, but the coefficient on $\overline{R_{t}-R_{m k t}}$ is somewhat closer to zero. Regression (3) shows that investors are also responsive to the information in Bayesian mixture alphas.

In regression (4), we see that both the OLS alphas and the Bayesian mixture alphas predict fund flows in a statistically significant manner. Although the point 
estimate of the coefficient on the Bayesian mixture alpha is higher than that of the OLS alpha, 0.33 versus 0.11 , this difference is not significant. The key finding is that the information contained in the Bayesian mixture alphas, incorporating knowledge of the skewness and excess kurtosis in the cross-sectional distribution of alpha, influences investors' capital allocations, beyond the information contained in OLS alphas and directly in past returns. Although not statistically significant, it also appears that including the Bayesian mixture alphas, in regressions (3) and (4), decreases the convexity of fund flows, consistent with the theoretical predictions of section 6.1.

Table 6.2 shows a set of related regressions where the lagged estimation residual from (2.1) is among the independent variables. By explicitly controlling for this "luck" component of returns as well as the "skill" component $\widehat{\alpha}$, we can examine investors' sensitivity to these components of returns. ${ }^{40}$ If investors were entirely rational, they would ignore the "luck" component of returns, but this is not what we find.

Regressions (6) and (7) show fund flow sensitivity to $\widehat{\alpha}$ and residuals from, respectively, OLS and Bayesian estimations. In both cases, investors respond very strongly to estimated residuals, even though this "luck" component is not persistent by construction. In the case of OLS estimates, the sensitivity to "luck" is an order of manitude larger than putative "skill" - $\widehat{\alpha}$, while for the Bayesian estimates the sensitivities are nearly identical.

\footnotetext{
${ }^{40}$ Systematic risk exposure through non-zero factor loadings also contributes to returns, but this component is not correlated with the "skill" and "luck" components and therefore doesn't need to be included as a regressor.
} 


\begin{tabular}{|c|c|c|c|c|}
\hline \multicolumn{5}{|c|}{$\begin{array}{l}\text { Fund Flow Predictability } \\
\text { Fama-MacBeth Regressions }\end{array}$} \\
\hline Flow Ratio & $(1)$ & $(2)$ & $(3)$ & $(4)$ \\
\hline Top Return Quintile & $\begin{array}{l}0.62^{*} \\
(0.17)\end{array}$ & $\begin{array}{l}0.50^{*} \\
(0.16)\end{array}$ & $\begin{array}{l}0.38^{*} \\
(0.16)\end{array}$ & $\begin{array}{l}0.43^{*} \\
(0.16)\end{array}$ \\
\hline Bottom Return Quintile & $\begin{array}{c}0.10 \\
(0.17)\end{array}$ & $\begin{array}{c}0.21 \\
(0.18)\end{array}$ & $\begin{array}{c}0.26 \\
(0.17)\end{array}$ & $\begin{array}{c}0.39 \\
(0.27)\end{array}$ \\
\hline$\overline{R_{t}-R_{m k t}}(\%)$ & $\begin{array}{l}2.45^{*} \\
(0.24)\end{array}$ & $\begin{array}{l}1.99^{*} \\
(0.27)\end{array}$ & $\begin{array}{l}2.14^{*} \\
(0.24)\end{array}$ & $\begin{array}{l}2.16^{*} \\
(0.35)\end{array}$ \\
\hline$\sigma\left(R_{t}-R_{m k t}\right)(\%)$ & $\begin{array}{l}-0.04 \\
(0.03)\end{array}$ & $\begin{array}{l}-0.04 \\
(0.04)\end{array}$ & $\begin{array}{l}-0.04 \\
(0.03)\end{array}$ & $\begin{array}{l}-0.06 \\
(0.04)\end{array}$ \\
\hline Lagged Expense Ratio (\%) & $\begin{array}{l}-0.15 \\
(0.18)\end{array}$ & $\begin{array}{l}-0.13 \\
(0.17)\end{array}$ & $\begin{array}{l}-0.11 \\
(0.18)\end{array}$ & $\begin{array}{l}-0.09 \\
(0.18)\end{array}$ \\
\hline Total Flow into Fund Category $(\$ M)$ & $\begin{array}{c}0.00 \\
(0.00)\end{array}$ & $\begin{array}{c}0.00 \\
(0.00)\end{array}$ & $\begin{array}{c}0.00 \\
(0.00)\end{array}$ & $\begin{array}{c}0.00 \\
(0.00)\end{array}$ \\
\hline Lagged Log(Assets Under Mgmt. (\$M)) & $\begin{array}{l}0.36^{*} \\
(0.05)\end{array}$ & $\begin{array}{r}-0.38^{*} \\
(0.05)\end{array}$ & $\begin{array}{c}-0.38^{*} \\
(0.05)\end{array}$ & $\begin{array}{c}-0.37^{*} \\
(0.05)\end{array}$ \\
\hline Lagged OLS $\widehat{\alpha}(\%)$ & & $\begin{array}{l}0.13^{*} \\
(0.02)\end{array}$ & & $\begin{array}{l}0.11^{*} \\
(0.05)\end{array}$ \\
\hline Lagged Bayesian Mixture $\widehat{\alpha}(\%)$ & & & $\begin{array}{c}0.65^{*} \\
(0.06)\end{array}$ & $\begin{array}{l}0.33^{*} \\
(0.14)\end{array}$ \\
\hline Constant & $\begin{array}{c}0.95 \\
(0.56)\end{array}$ & $\begin{array}{l}1.35^{*} \\
(0.58)\end{array}$ & $\begin{array}{l}1.67^{*} \\
(0.60)\end{array}$ & $\begin{array}{c}1.40^{*} \\
(0.64)\end{array}$ \\
\hline$R^{2}$ & 0.09 & 0.09 & 0.09 & 0.10 \\
\hline
\end{tabular}

Table 6.1 - Results for Fama-MacBeth regressions predicting flow ratio, defined as this month's net flows divided by last month's total assets under management; 1963-2010. Regression (1) is based directly on the baseline regression in Sirri and Tufano (1998), and shows both the responsiveness of flows to raw past performance, as well as the convexity of fund flows. (2) adds OLS alphas as a predictor, while (3) adds the Bayesian mixture alphas. (4) includes both alphas as predictors. Although overall $R^{2}$ does not change dramatically, the strongly significant coefficients on the Bayesian mixture alphas indicate that they contain information not contained in the other predictors, and that investors are sensitive to this information. 
Fund Flow Predictability with "Skill" and "Luck" Decomposition Fama-MacBeth Regressions

\begin{tabular}{|c|c|c|c|}
\hline Flow Ratio & $(5)$ & (6) & $(7)$ \\
\hline Top Return Quintile & $\begin{array}{l}2.18^{*} \\
(0.09)\end{array}$ & $\begin{array}{l}1.36^{*} \\
(0.08)\end{array}$ & $\begin{array}{l}1.45^{*} \\
(0.08)\end{array}$ \\
\hline Bottom Return Quintile & $\begin{array}{c}-1.66^{*} \\
(0.07)\end{array}$ & $\begin{array}{c}-0.92^{*} \\
(0.07)\end{array}$ & $\begin{array}{c}-1.05^{*} \\
(0.06)\end{array}$ \\
\hline$\sigma\left(R_{t}-R_{m k t}\right)(\%)$ & $\begin{array}{c}0.03^{*} \\
(0.01)\end{array}$ & $\begin{array}{c}-0.01 \\
(0.02)\end{array}$ & $\begin{array}{l}-0.01 \\
(0.02)\end{array}$ \\
\hline Lagged Expense Ratio (\%) & $\begin{array}{c}-0.06 \\
(0.11)\end{array}$ & $\begin{array}{c}-0.02 \\
(0.12)\end{array}$ & $\begin{array}{l}-0.02 \\
(0.12)\end{array}$ \\
\hline Total Flow into Fund Category $(\$ M)$ & $\begin{array}{c}0.00 \\
(0.00)\end{array}$ & $\begin{array}{c}0.00 \\
(0.00)\end{array}$ & $\begin{array}{c}0.00 \\
(0.00)\end{array}$ \\
\hline Lagged Log(Assets Under Mgmt. (\$M)) & $\begin{array}{c}-0.35^{*} \\
(0.03)\end{array}$ & $\begin{array}{c}-0.39^{*} \\
(0.03)\end{array}$ & $\begin{array}{c}-0.40^{*} \\
(0.03)\end{array}$ \\
\hline Lagged OLS $\widehat{\alpha}(\%)$ & & $\begin{array}{l}1.41^{*} \\
(0.30)\end{array}$ & \\
\hline Lagged OLS "Luck" (\%) & & $\begin{array}{c}14.46^{*} \\
(3.61)\end{array}$ & \\
\hline Lagged Bayesian Mixture $\widehat{\alpha}(\%)$ & & & $\begin{array}{c}16.80^{*} \\
(3.98)\end{array}$ \\
\hline Lagged Bayesian Mixture "Luck" (\%) & & & $\begin{array}{c}15.90^{*} \\
(4.00)\end{array}$ \\
\hline Constant & $\begin{array}{l}2.13^{*} \\
(0.26)\end{array}$ & $\begin{array}{l}2.64^{*} \\
(0.25)\end{array}$ & $\begin{array}{l}3.07^{*} \\
(0.26)\end{array}$ \\
\hline$R^{2}$ & 0.07 & 0.10 & 0.09 \\
\hline
\end{tabular}

Table 6.2 - Results for Fama-MacBeth regressions predicting flow ratio using "skill" and "luck" decomposition; 1963-2010. Regression (5) presents a baseline model similar to regression (1) in Table 6.1 except that mean excess return has been removed as a predictor. Regresssion (6) adds both OLS $\widehat{\alpha}-$ "skill" — and the previous month's estimation residual from equation (2.1) — "luck". Regression (7) does the same for the Bayesian "skill" and "luck" components. 


\subsection{Smart money effect}

A general finding in the literature is that mutual fund flows help to predict mutual fund performance. ${ }^{41}$ The "smart money" effect refers to the interpretation that investors are actually able to anticipate future fund performance and allocate capital accordingly, but this is not the only interpretation. ${ }^{42}$ One way to assess the "smartness" of investors is to examine the incremental impact that estimated alphas have for fund return prediction, controlling for fund flows.

Table 6.3 presents estimates from a series of regressions seeking to explain fund returns in terms of past flows and returns. Regression (3) shows that past flows do predict fund returns; adding past returns in regression (4) weakens the predictive power of flows. As expected, regressions (1) and (2) show that including lagged OLS and Bayesian mixture alphas does improve fund return prediction.

The most interesting results are from regression (4), which includes past flows and both OLS and Bayesian mixture alphas. The coefficient on lagged flow ratio remains significant-flows are predictive beyond the information contained in estimated alphas. Had the coefficients on lagged flows all been insignificant, this would have implied that alphas predict fund returns because both are driven by managerial skill: flows are truly smart money. The failure of this test does not imply that investors are not smart, however, since this is a joint test of the accuracy of the estimated alphas and the smart money effect. But the strength of the return predictability documented in section 4.4 and the relative weakness of the flow predictability documented in section 6.2 suggest a mechanism other than "smart money" for the predictability of fund returns by fund flows.

\footnotetext{
${ }^{41}$ See, e.g., Gruber (1996) and Zheng (1999).

${ }^{42}$ For example, Lou (2011) presents evidence that fund return predictability is the consequence of flow-induced price pressure on underlying stock holdings.
} 


\begin{tabular}{cccccc}
\hline \multicolumn{5}{c}{ "Smart Money" Effect, Fama-MacBeth Regressions } \\
\hline Monthly Fund Return $(\%)$ & $(1)$ & $(2)$ & $(3)$ & $(4)$ & $(5)$ \\
\hline Lagged OLS $\widehat{\alpha}(\%)$ & $0.19^{*}$ & & & & $-0.15^{*}$ \\
& $(0.01)$ & & & & $(0.01)$ \\
Lagged Bayesian Mixture $\widehat{\alpha}(\%)$ & & $0.11^{*}$ & & & $0.11^{*}$ \\
& & $(0.01)$ & & & $(0.01)$ \\
& & & & $0.24^{*}$ & $0.17^{*}$ \\
$R_{t}-R_{m k t}(\%)$ & & & $(0.01)$ & $(0.01)$ \\
& & & $0.002^{*}$ & $0.001^{*}$ & $0.001^{*}$ \\
Flow Ratio in month $t_{-1}$ & & & $(0.00)$ & $(0.00)$ & $(0.00)$ \\
& & & $0.001^{*}$ & 0.00 & 0.00 \\
Flow Ratio in month $t_{-2}$ & & & $(0.00)$ & $(0.00)$ & $(0.00)$ \\
& & & 0.00 & 0.00 & 0.00 \\
Flow Ratio in month $t_{-3}$ & & & $(0.00)$ & $(0.00)$ & $(0.00)$ \\
& & & & & \\
Constant & $0.45^{*}$ & $0.53^{*}$ & $0.49^{*}$ & $0.38^{*}$ & $0.49^{*}$ \\
& $(0.09)$ & $(0.09)$ & $(0.01)$ & $(0.01)$ & $(0.01)$ \\
\hline$R^{2}$ & 0.02 & 0.02 & 0.01 & 0.02 & 0.02 \\
\hline
\end{tabular}

Table 6.3 - Results for Fama-MacBeth regressions predicting monthly fund returns; 1963-2010. (1) and (2) show the predictive power of OLS and Bayesian mixture alphas, respectively. (3) shows that flows do help to predict fund performance. They continue to predict performance when (4) past returns are included, and (5) when OLS and Bayesian mixture alphas are included. This evidence, combined with the results from sections 4.4 and 6.2 , suggests that "smart money" is not the principal mechanism explaining why fund flows predict fund returns.

Also noteworthy is the coefficient on lagged OLS alphas in regression (5): it is negative and significant. Controlling for Bayesian mixture alphas, future fund returns are higher when OLS alphas are lower, and vice versa. This is a clear illustration of how poorly OLS distinguishes signal from noise in the context of investment performance evaluation. 


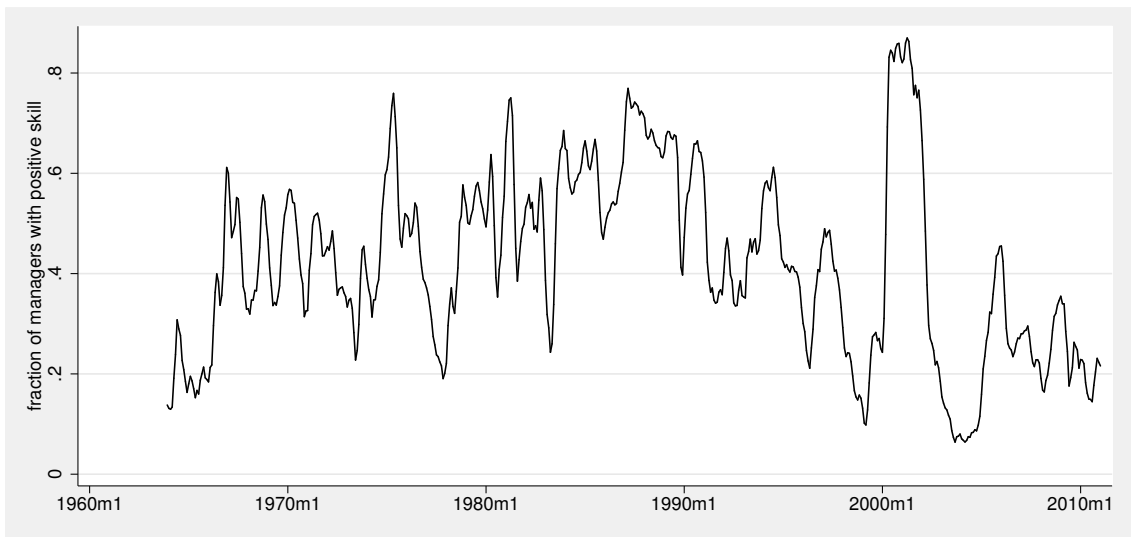

Figure 6.2 - Estimated fraction of managers with positive alpha using the Bayesian mixture model.

\subsection{Value of active management}

Even if some fund managers possess skill, investors do not benefit unless managers generate positive alpha after management fees and trading costs. Since all alphas in this study are estimated using net fund returns to the investor, we can directly evaluate the prevalence of skill over time. Panel A of Figure 4.3 shows the overall mean of the prevailing distribution of skill — it does not diverge substantially from the implied OLS mean - which seems to lend support to the Berk and Green hypothesis: the typical fund has an alpha quite close to zero.

Looking at the mean alone is misleading, however. Figure 4.4 shows that the prevailing distribution of skill also has fat tails and positive skewness. Instead of simply examining the mean, it is more informative to compute the fraction of managers with positive alpha directly from the estimated parameters of the prevailing cross-sectional distribution. The results are shown in Figure 6.2. 
Barras et al. (2010) classify funds as either unskilled, zero-alpha, or skilled, and find that $75 \%$ of funds are zero-alpha while less than $5 \%$ are skilled. ${ }^{43}$ This paper uses fund-level data to estimate the underlying distribution-sharply separating the negative and positive regions of skill. There is no need for a zero-alpha category unless there is a point mass at zero in the underlying skill distribution. Kosowski et al. (2006) use a bootstrap approach to compute a continuous measure of skill, and find that approximately $5 \%$ of managers show evidence of skill net of costs.

The most recent estimates in Figure 6.2 suggest that approximately $20 \%$ of managers have skill, although the fraction has been much higher in the past. The volatility of the estimates over time and the magnitude of the maximum estimates rightly suggest that the distribution has not been perfectly estimated. Nevertheless, the decile portfolios performed well: an imperfectly estimated distribution still greatly assists in the proper ordering of funds.

Even if sizeable fractions of managers possess skill after costs and fees, we would like to know if the active mutual fund industry is providing value in aggregate. Fama and French (2010) employ a bootstrap methodology and find that the aggregate portfolio of mutual funds has a negative net alpha, although there is evidence of superior skill in the extreme upper tail of the cross-sectional distribution of skill.

This study used only one representative share class from each fund. To construct a comparable net industry-wide alpha, we would need to take into account all share classes of each fund, their expense ratios, and assets under management, following French (2008). This has not been done, so the important question of whether the industry provides value to investors remains open. Even still, it is safe to speculate

\footnotetext{
${ }^{43}$ See Figure 4 in Barras et al. (2010) for a comparison to Figure 6.2. Their estimated fraction of skill is both much lower and smoother than the estimates presented here.
} 
that such an analysis would have conclusions somewhat more favorable to the mutual fund industry than French, given the higher fraction of skilled managers found here. 


\section{Section 7}

\section{Conclusion}

The model explored in this paper is deliberately kept simple to focus attention on the sensitivity of alpha estimates to the assumptions made regarding the cross-section of skill. Existing methodologies ignore the conclusions of the "superstars" literature: the returns to investing skill are likely to be positively skewed and fat-tailed. An optimal approach to performance evaluation should take this into account and explicitly model the cross-sectional distribution of alpha accordingly.

The hierarchical model I propose is such an approach. While it does not impose skewness or excess kurtosis, it "learns" from the data that such features are indeed present. Despite the simplicity of the mixture-of-normals distribution, the resulting estimates for fund alphas exhibit strong predictability across the entire population of funds - it is not merely the very best and the very worst funds whose performance persists.

In addition to accurately measuring fund alphas, this model also provides a possible explanation for the convexity of fund flows. Any non-normal prior on the distribution of alpha will lead to a nonlinear updating rule: high observed returns can exert much greater influence than low observed returns on the posterior mean of a given fund's alpha. The convexity of fund flows could thus be a rational response to the non-normality of alpha. 


\section{Part IV}

End Matter 


\section{Appendix A}

\section{MCMC Algorithm: Mixture of Normals}

This section provides details about the Bayesian inference procedure, including the priors on all parameters and a step-by-step summary of the Markov chain Monte Carlo (MCMC) algorithm used to generate samples from the joint posterior distribution of the parameters when alphas are assumed to be draw from a finite mixture of normals.

\section{A.1 Parameters to be estimated}

$\left(\mu_{i}, V_{i}, w_{i}, \alpha^{j}, \beta_{k}^{j}, V_{\varepsilon}^{j}\right)$ for $i=1, \ldots, N ; j=1, \ldots, J ; k=1, \ldots, K$; where $N$ is the number of mixture components in (2.2), $J$ is the number of funds, and $K$ is the number of risk factors in $(2.1)$.

\section{A.2 Latent indicator}

To simplify the estimation procedure, I introduce a latent $J \times 1$ vector $z$, where each element is Bernoulli distributed, ${ }^{44}$

$$
z_{j} \sim \operatorname{Bernoulli}\left(w_{2}\right)
$$

\footnotetext{
${ }^{44}$ In the general case with more than two mixture components, $z_{j}$ is categorically distributed with vector parameter $w$.
} 
The conditional (on $z_{j}$ ) prior on skill is now normal, instead of the original mixture of normals in (2.2):

$$
\alpha^{j} \sim N\left(\mu_{z_{j}+1}, V_{z_{j}+1}\right)
$$

\section{A.3 Priors}

All priors are proper but diffuse. They are scaled to correspond to the monthly percent returns used in the analysis. For example, an annual alpha of $12 \%$ would appear here as 1 .

$$
\begin{gathered}
\mu_{1} \sim N\left(a_{\mu}^{\text {prior }}=0, A_{\mu}^{\text {prior }}=10\right) \\
\mu_{2} \sim N\left(a_{\mu}^{\text {prior }}=0, A_{\mu}^{\text {prior }}=10\right) \cdot \mathbf{1}_{\mu_{2} \geq \mu_{1}} \\
V_{i} \sim \operatorname{Gamma}(1,10) \\
w \sim \text { Dirichlet }\left(\pi^{\text {prior }}=\left(\begin{array}{l}
1 \\
1
\end{array}\right)\right) \\
\beta_{m k t}^{j} \sim N\left(a_{\beta_{\text {mkt }}}^{\text {prior }}=1, A_{\beta}^{\text {prior }}=5\right) \\
\beta_{k}^{j} \sim N\left(a_{\beta}^{\text {prior }}=0, A_{\beta}^{\text {prior }}=5\right) \\
V_{\varepsilon}^{j} \sim \operatorname{Gamma}(1,5)
\end{gathered}
$$

\section{A.4 Sampling algorithm}

1. Draw $z$ conditional on $w, \mu_{i}, V_{i}, \alpha$.

- Compute (vector) probabilities $\omega$ conditional on each mixture component:

$$
\omega_{i}=\frac{1}{\sqrt{V_{i}}} \cdot \phi\left(\frac{\alpha-\mu_{i}}{\sqrt{V_{i}}}\right) .
$$


- Compute (vector) unconditional posterior probability:

$$
p_{2}=\frac{\omega_{2}}{\omega_{1}+\omega_{2}}
$$

- Draw $z \sim \operatorname{Bernoulli}\left(p_{2}\right)$.

2. Partition $\alpha$ conditional on $z$.

- Partition $\alpha$ into two vectors, $\alpha_{1}$ and $\alpha_{2}$, corresponding to those elements of $\alpha$ from each of the mixture components, and let $N_{i}=\operatorname{rows}\left(\alpha_{i}\right)$.

3. Draw $V_{i}$ conditional on $z, \mu_{i}, \alpha_{i}$.

- Because of the gamma prior, the posterior on $V_{i}$ is not a known distribution and is sampled using a random walk Metropolis-Hastings draw.

4. Draw $\mu_{i}$ conditional on $V_{i}, \alpha_{i}$.

- Compute posterior hyper-parameters for each mixture component:

$$
\begin{gathered}
A_{\mu, i}^{\text {posterior }}=\left(\frac{1}{A_{\mu}^{\text {prior }}}+\frac{N_{i}}{V_{i}}\right)^{-1} \\
\frac{a_{\mu, i}^{\text {posterior }}}{A_{\mu, i}^{\text {posterior }}}=\frac{a_{\mu}^{\text {prior }}}{A_{\mu}^{\text {prior }}}+\frac{\sum_{n=1}^{N_{i}} \alpha_{i, n}}{V_{i}}
\end{gathered}
$$

- Draw $\mu_{i} \sim N\left(a_{\mu, i}^{\text {posterior }}, A_{\mu, i}^{\text {posterior }}\right)$.

5. Impose identifying constraint $\mu_{2} \geq \mu_{1}$.

- If the most recent draws of $\mu_{i}$ violate this constraint, revert to the previous values. 
6. Draw $w$ conditional on $z$.

- Compute posterior vector hyper-parameter:

$$
\pi^{\text {posterior }}=\pi^{\text {prior }}+\left(\begin{array}{c}
N_{1} \\
N_{2}
\end{array}\right)
$$

- Draw $w \sim \operatorname{Dirichlet}\left(\pi^{\text {posterior }}\right)$.

7. Draw $V_{\varepsilon}^{j}$ conditional on $R^{j}, F, \alpha^{j}, \beta^{j}$.

- Because of the gamma prior, the posterior on $V_{\varepsilon}^{j}$ is not a known distribution and is sampled using a random walk Metropolis-Hastings draw.

8. Draw $\beta^{j}$ conditional on $R^{j}, F, \alpha^{j}, V_{\varepsilon}^{j}$

- Compute posterior hyper-parameters for each $\beta_{k}^{j}$ :

$$
\begin{gathered}
A_{\beta, j, k}^{\text {posterior }}=\left(\frac{1}{A_{\beta, k}^{\text {prior }}}+\frac{F_{k}^{\prime} \cdot F_{k}}{V_{\varepsilon}^{j}}\right)^{-1} \\
\frac{a_{\beta, j, k}^{\text {posterior }}}{A_{\beta, j, k}^{\text {posterior }}}=\frac{a_{\beta, k}^{\text {prior }}}{A_{\beta, k}^{\text {prior }}}+\frac{F_{k}^{\prime} \cdot R^{j}-\alpha^{j} \cdot \sum_{t=1}^{T} F_{k, t}-F_{k}^{\prime} \cdot F_{-k} \cdot \beta_{-k}}{V_{\varepsilon}^{j}}
\end{gathered}
$$

- Draw $\beta_{k}^{j} \sim N\left(a_{\beta, j, k}^{\text {posterior }}, A_{\beta, j, k}^{\text {posterior }}\right)$.

9. Draw $\alpha^{j}$ conditional on $z^{j}, \mu_{z^{j}+1}, V_{z^{j}+1}, \beta^{j}, V_{\varepsilon}^{j}, F, R^{j}$.

- Compute posterior hyper-parameters for each $\alpha^{j}$ :

$$
A_{\alpha, j}^{\text {posterior }}=\left(\frac{1}{V_{z^{j}+1}}+\frac{T}{V_{\varepsilon}^{j}}\right)^{-1}
$$




$$
\frac{a_{\alpha, j}^{\text {posterior }}}{A_{\alpha, j}^{\text {posterior }}}=\frac{\mu_{z^{j}+1}}{V_{z^{j}+1}}+\frac{\sum_{t=1}^{T}\left(R_{t}^{j}-F_{t} \cdot \beta^{j}\right)}{V_{\varepsilon}^{j}}
$$

- Draw $\alpha^{j} \sim N\left(a_{\alpha, j}^{\text {posterior }}, A_{\alpha, j}^{\text {posterior }}\right)$. 


\section{Appendix B}

\section{Moments of the Generalized Unimodal Dis-}

\section{tribution}

The mode of the generalized unimodal distribution of Fernández and Steel (1998) in (5.1) is $\mu$. To better understand this distribution, it is helpful to examine its moments as well.

Equation (5.1) generates a finite $r^{\text {th }}$ order moment if and only if the corresponding moment of the underlying Student's $t$-distribution exists. In general, the $r^{\text {th }}$ central moment of (5.1) is given by

$$
E\left[\left(\alpha^{j}\right)^{r} \mid \gamma\right]=M_{r} \cdot \frac{\gamma^{r+1}+\frac{(-1)^{r}}{\gamma^{r+1}}}{\gamma+\frac{1}{\gamma}}
$$

where $M_{r}$ is defined with respect to the Student's $t$-distribution in (5.2):

$$
M_{r}=\int_{\mu}^{\infty}(s-\mu)^{r} \cdot \frac{2}{\sqrt{V}} \cdot f_{\nu}\left(\frac{s-\mu}{\sqrt{V}}\right) \mathrm{d} s
$$

The skewness of the distribution in (5.1) is given by

$$
\operatorname{Skew}\left(\alpha^{j} \mid \gamma\right)=\left(\gamma-\frac{1}{\gamma}\right) \cdot \frac{\left(M_{3}+2 M_{1}^{3}-3 M_{1} M_{2}\right)\left(\gamma^{2}+\frac{1}{\gamma^{2}}\right)+3 M_{1} M_{2}-4 M_{1}^{3}}{\left[\left(M_{2}-M_{1}^{2}\right)\left(\gamma^{2}+\frac{1}{\gamma^{2}}\right)+2 M_{1}^{2}-M_{2}\right]^{\frac{3}{2}}} .
$$




\section{Bibliography}

[1] Baks, Klaas P., Andrew Metrick, and Jessica Wachter, 2001, Should Investors Avoid All Actively Managed Mutual Funds? A Study in Bayesian Performance Evaluation, Journal of Finance 56, 45-85.

[2] Barras, Laurent, Oliver Scaillet, and Russ Wermers, 2010, False Discoveries in Mutual Fund Performance: Measuring Luck in Estimated Alphas, Journal of Finance 65, 179-216.

[3] Berk, Jonathan B. and Richard C. Green, 2004, Mutual Fund Flows and Performance in Rational Markets, Journal of Political Economy 112, 1269-1295.

[4] Bollen, Nicolas P. B. and Jeffrey A. Busse, 2004, Short-Term Persistence in Mutual Fund Performance, Review of Financial Studies 18, 569-597.

[5] Brown, David P. and Youchang Wu, Mutual Fund Families and Performance Evaluation (February 15, 2011). Available at SSRN: http://ssrn.com/abstract $=1690539$

[6] Busse, Jeffrey A. and Paul J. Irvine, 2006, Bayesian Alphas and Mutual Fund Persistence, Journal of Finance 61, 2251-2288.

[7] Carhart, Mark M., 1997, On Persistence in Mutual Fund Performance, Journal of Finance 52, 57-82.

[8] Chevalier, Judith and Glenn Ellison, 1997, Risk Taking by Mutual Funds as a Response to Incentives, Journal of Political Economy 105, 1167-1200.

[9] Chevalier, Judith and Glenn Ellison, 1999, Are Some Mutual Fund Managers Better Than Others? Cross-Sectional Patterns in Behavior and Performance, Journal of Finance 54, 875-899.

[10] Chung, Kee H. and Ramond A. K. Cox, 1990, Patterns of Productivity in the Finance Literature: A Study of the Bibliometric Distributions, Journal of Finance 45, 301-309.

[11] Cohen, Lauren, Andrea Frazzini, and Christopher Malloy, 2008, The Small World of Investing: Board Connections and Mutual Fund Returns, Journal of Political Economy 116, 951-979. 
[12] Coval, Joshua D. and Tobias J. Moskowitz, 2001, The Geography of Investment: Informed Trading and Asset Prices, Journal of Political Economy 109, 811-841.

[13] Efron, Bradley and Carl Morris, 1977, Stein's Paradox in Statistics, Scientific American 236, 119-127.

[14] Evans, Richard B., 2010, Mutual Fund Incubation, Journal of Finance 65, 15811611.

[15] Fama, Eugene F. and Kenneth R. French, 1993, Common risk factors in the returns on bonds and stocks, Journal of Financial Economics 33, 3-53.

[16] Fama, Eugene F. and Kenneth R. French, 2010, Luck versus Skill in the CrossSection of Mutual Fund Returns, Journal of Finance 65, 1915-1947.

[17] Fernández, Carmen and Mark F. J. Steel, 1998, On Bayesian Modeling of Fat Tails and Skewness, Journal of the American Statistical Association 93, 359-371.

[18] French, Kenneth R., 2008, Presidential Address: The Cost of Active Investing, Journal of Finance 63, 1537-1573.

[19] Gabaix, Xavier and Augustin Landier, 2008, Why Has CEO Pay Increased So Much?, Quarterly Journal of Economics 123, 49-100.

[20] Gelman, Andrew, 2006, Prior distributions for variance parameters in hierarchical models, Bayesian Analysis 1, 515-533.

[21] Gruber, Martin J., 1996, The Growth in Actively Managed Funds, Journal of Finance 51, 783-810.

[22] Hausman, Jerry A. and Gregory K. Leonard, 1997, Superstars in the National Basketball Association: Economic Value and Policy, Journal of Labor Economics $15,586-624$.

[23] Jones, Christopher S. and Jay Shanken, 2005, Mutual fund performance with learning across funds, Journal of Financial Economics 78, 507-552.

[24] Kosowski, Robert, Allan Timmerman, Russ Wermers, and Hal White, 2006, Can Mutual Fund "Stars" Really Pick Stocks? New Evidence from a Bootstrap Analysis, Journal of Finance 61, 2551-2595.

[25] Krueger, Alan B., 2005, The Economics of Real Superstars: The Market for Rock Concerts in the Material World, Journal of Labor Economics 23, 1-30.

[26] Leamer, Edward E., 1978, Specification Searches.

[27] Levenshtein, V. I., 1966, Binary Codes Capable of Correcting Deletions, Insertions, and Reversals, Soviet Physics Doklady 10, 707-710. 
[28] Lindley, D. V., 1972, Bayesian Statistics, A Review.

[29] Lou, Dong, 2011, A Flow-Based Explanation for Return Predictability, working paper.

[30] Pástor, Ľuboš and Robert F. Stambaugh, 2002, Mutual fund performance and seemingly unrelated assets, Journal of Financial Economics 63, 315-349.

[31] Lynch, Anthony W. and David K. Musto, 2003, How Investors Interpret Past Fund Returns, Journal of Finance 58, 2033-2058.

[32] Mamaysky, Harry, Matthew Spiegel, and Hong Zhang, 2007, Improved Forecasting of Mutual Fund Alphas and Betas, Review of Finance 11, 359-400.

[33] Massa, Massimo, Jonathan Reuter, and Eric Zitzewitz, 2010, When should firms share credit with employees? Evidence from anonymously managed mutual funds, Journal of Financial Economics 95, 400-424.

[34] Reuter, Jonathan and Eric Zitzewitz, 2010, How Much Does Size Erode Mutual Fund Performance? A Regression Discontinuity Approach, NBER Working Paper 16329.

[35] Robert, Christian P. and George Casella, 2004, Monte Carlo Statistical Methods.

[36] Rosen, Sherwin, 1981, The Economics of Superstars, American Economic Review $71,845-858$.

[37] Sirri, Erik R. and Peter Tufano, 1998, Costly Search and Mutual Fund Flows, Journal of Finance 53, 1589-1622.

[38] Stephens, Matthew, 2000, Dealing with label switching in mixture models, Journal of the Royal Statistical Society B 62, 795-809.

[39] Wermers, Russ, 2000, Mutual Fund Performance: An Empirical Decomposition into Stock-Picking Talent, Style, Transactions Costs, and Expenses, Journal of Finance 55, 1655-1695.

[40] Zheng, Lu, 1999, Is Money Smart? A Study of Mutual Fund Investors' Fund Selection Ability, Journal of Finance 54, 901-933. 